\title{
On the difference equations with
}

\section{periodic coefficients}

\author{
V.Buslaev, A.Fedotov \\ Department of Mathematics and Mathematical Physics, St.Petersburg State \\ University, 1, Ulianovskaja, St.Petersburg-Petrodvorets Russia \\ buslaev@mph.phys.spbu.ru, fedotov@mph.phys.spbu.ru
}

\begin{abstract}
In the paper, we study entire solutions of the difference equation $\psi(z+h)=$ $M(z) \psi(z), z \in \mathbb{C}, \psi(z) \in \mathbb{C}^{2}$. In this equation, $h$ is a fixed positive parameter, and $M: \mathbb{C} \mapsto S L(2, \mathbb{C})$ is a given matrix function. We assume that $M(z)$ is a $2 \pi$-periodic trigonometric polynomial. The main aim is to construct the minimal entire solutions, e.i. the solutions with the minimal possible growth simultaneously as for $z \rightarrow-i \infty$ so for $z \rightarrow+i \infty$.

We show that the monodromy matrices corresponding to the bases made of the minimal solutions are trigonometric polynomials of the same order as the matrix $M$. This property relates the spectral analysis of the one dimensional difference Schrödinger equations with the potentials being trigonometric polynomials to an analysis of a finite dimensinal dynamical system.
\end{abstract}

\section{Introduction: Some definitions and main results}

The paper is devoted to the study of entire solutions of the equation

$$
\psi(z+h)=M(z) \psi(z), \quad z \in \mathbb{C}, \quad \psi(z) \in \mathbb{C}^{2} .
$$

In this equation, $h$ is a fixed positive parameter, and $M$ is a given matrix. We assume that the matrix $M$ satisfies the following two conditions. First,

$$
M(z) \in S L(2, \mathbb{C}), \quad z \in \mathbb{C},
$$

secondly, the matrix $M$ is a $2 \pi$-periodic trigonometric polynomial.

e-print archive: http://xxx.lanl.gov/hep-th/98111131 
Since equation (1) is invariant with respect to the multiplication of the solutions by the $h$-periodic functions, one can construct its entire solutions growing as for $z \rightarrow-i \infty$ so for $z \rightarrow+i \infty$ as quickly as wanted. The main aim of this paper is to construct the minimal entire solutions, e.i. the solutions with the minimal possible growth simultaneously as for $z \rightarrow-i \infty$ so for $z \rightarrow+i \infty$.

The set of solutions of $(1)$ is invariant with respect to the operator of $2 \pi$-translation: $f(z) \mapsto f(z+2 \pi)$, and there are natural objects, monodromy matrices, describing the transformations of the bases in the space of solutions of (1) under such translations, see subsection 1.10. The notion of the monodromy matrix for difference equations with periodic coefficients is a natural generalization of the classical notion of the monodromy matrix for ordinary differential equations with periodic coefficients. For the differential equations, the monodromy matirces are constant, i.e. independent of the variable of the equation. For the difference equations, the monodromy matrices are periodic functions of the variable. We show that the monodromy matrices corresponding to the bases made of the minimal solutions have the most simple analytic structure: they are trigonometric polynomials of the same order as the matrix $M$.

The monodromy matrices appear in the spectral analysis of one dimensinal Schrödinger equations with periodic potentials. The spectral analysis of a differential Schrödinger equation with a periodic potential reduces to the study of the spectrum of a (constant) monodromy matrix. This leads a simple "discret" band structure of the spectra of the periodic differential equations. For difference equations, the monodromy matrices being periodic, instead of the spectral analysis of an individual monodromy matrix, one arrives to an infinite sequence of the monodromy matrices and has to study properties of an infinite sequence of finite difference equations of the form (1). This reflects the cantorien structure of the spectra of difference equations with periodic coefficients. The above property of the monodromy matrices corresponding to the minimal entire solutions is important for the spectral analysis of the difference Schrödinger equations with the potentials being trigonometric polynomials. It relates their spectral analysis to an analysis of a finite dimensinal dynamical system.

This paper is a natural continuation of the articles [7] - [5] devoted to Harper equation

$$
\frac{\psi(z+h)+\psi(z-h)}{2}+\cos z \psi(z)=E \psi(z)
$$

and was inspirated by the papers of B.Helffer and J.Sjöstrand, and of Wilkinson (see, for example, $[1,10])$. They have suggested an asymptotic renormalization method to study the spectrum of the Harper equation (2). Our works are related to an exact renormalization procedure, the monodromization method. 


\subsection{Notations and agreements}

1. To simplify the notations, we let

$$
a(z)=M_{11}(z), \quad b(z)=M_{12}(z), \quad c(z)=M_{21}(z), \quad d(z)=M_{22}(z) .
$$

2. If either $b(z) \equiv 0$, or $c(z) \equiv 0$, then the vector equation (1) reduces to two scalar equations which can be solved explicitly. In this paper, we always assume that

$$
b(z) \not \equiv 0,
$$

and let

$$
\rho(z)=b(z) / b(z-h), \quad v(z)=a(z)+\rho(z) d(z-h) .
$$

These two functions play an essential part in our constructions. Elementary calculations show that if $\psi$ is a vector solution of (1), then its first component satisfies the equation

$$
\psi_{1}(z+h)+\rho(z) \psi_{1}(z-h)=v(z) \psi_{1}(z)
$$

3. When describing the vector solutions of (1), we describe only their first components: if the first component of a vector solution is known, the second one can be reconstructed by the formula

$$
\psi_{2}(z)=\frac{\psi_{1}(z+h)-a(z) \psi_{1}(z)}{b(z)} .
$$

4. Throughout the paper, we use also the following notations. Let $f$ be a $2 \pi$ periodic function. If

$$
f(z)=f_{-m} e^{-i m z}(1+o(1)), \quad f_{-m} \neq 0, \quad z \rightarrow+i \infty
$$

we put

$$
n_{+}(f)=m, \quad f_{+}=f_{-m}
$$

and if

$$
f(z)=f_{l} e^{i l z}(1+o(1)), \quad f_{l} \neq 0, \quad z \rightarrow-i \infty
$$

we let

$$
n_{-}(f)=l, \quad f_{-}=f_{l}
$$

\subsection{Set of analytic solutions of (1)}

List some elementary properties of the set of analytic solutions of equation (1), see, for example, [5]. 
Fix $\alpha, \beta \in \mathbb{R}, \alpha<\beta$. Let $S$ be the strip

$$
S=\{z \in \mathbb{C}: \alpha<\operatorname{Im} z<\beta\}
$$

and let $\mathbb{M}(S)$ be the set of the vector solutions of (1) analytic in this strip. Denote the ring of all $h$-periodic functions analytic in $S$ by $\mathbb{K}(S)$.

For any two solutions $\psi_{1}, \psi_{2} \in \mathbb{M}(S)$ the wronskian

$$
\left\{\psi_{1}(z), \psi_{2}(z)\right\} \equiv \operatorname{det}\left(\psi_{1}(z), \psi_{2}(z)\right)
$$

is an element of $\mathbb{K}(S)$. The solutions $\psi_{1}, \psi_{2}$ are linearly independent over the ring $\mathbb{K}(S)$ if $\left\{\psi_{1}(z), \psi_{2}(z)\right\} \neq 0, z \in S$.

Let two solutions $\psi_{1}, \psi_{2} \in \mathbb{M}(S)$ be linearly independent over the ring $\mathbb{K}(S)$. Then any solution $\psi \in \mathbb{M}(S)$ can be uniquely represented in the form

$$
\psi=\alpha \psi_{1}+\beta \psi_{2}, \quad \alpha, \beta \in \mathbb{K}(S) .
$$

Thus, the set $\mathbb{M}(S)$ is a two dimensional module over the ring $\mathbb{K}(S)$, and the solutions $\psi_{1}, \psi_{2}$ form a basis of $\mathbb{M}(S)$.

In the case of $S=\mathbb{C}$, we write simply $\mathbb{M}$ and $\mathbb{K}$.

\subsection{Bloch solutions}

The set $\mathbb{M}(S)$ is invariant with respect to the $2 \pi$-translations. We call $f \in \mathbb{M}(S)$ a Bloch solution if

$$
f(z+2 \pi)=u(z) f(z), \quad u \in \mathbb{K}(S) .
$$

The factor $u$ is called the Bloch multiplier of the solution $f$. Bloch solutions play an important role in the theory of (1).

\subsection{Singular points of equation (1)}

The $2 \pi$-periodicity of the matrix $M$ makes natural to consider $+i \infty$ and $-i \infty$ as two singular "points" of the equation. For any fixed $Y \in \mathbb{R}$, call the halfplane $\mathbb{C}_{+}=\mathbb{C}_{+}(Y)=\{z \in \mathbb{C}: \operatorname{Im} z>Y\}$ a vicinity of $+i \infty$, and the half-plane $\mathbb{C}_{-}=\mathbb{C}_{-}(Y)=\{z \in \mathbb{C}: \operatorname{Im} z<Y\}$ a vicinity of the $-i \infty$. For brevity, we shall write

$$
\mathbb{M}_{ \pm}=\mathbb{M}\left(\mathbb{C}_{ \pm}\right), \quad \mathbb{K}_{ \pm}=\mathbb{K}\left(\mathbb{C}_{ \pm}\right)
$$

The minimal entire solutions of equation (1) can be characterized as the entire solutions having the "simplest" asymptotic behavior as for $z \rightarrow-i \infty$ so for

$z \rightarrow+i \infty$. 


\subsection{Canonical bases in vicinities of the singular points}

We shall define the minimal solutions in terms of the "simplest" solutions living and analytic in some vicinities of the singular points $\pm i \infty$. They appear to be Bloch solutions. One has

Theorem 1.1a. Let $b \not \equiv 0$, and let $v \rightarrow \infty$ as $z \rightarrow+i \infty$. There exist two vector Bloch solutions $f_{1,2}$ of (1) analytic in a vicinity $\mathbb{C}_{+}$of $+i \infty$ with the first components admitting the representations

$$
\left(f_{1,2}\right)_{1}(z)=e^{ \pm \frac{i}{2 h n_{+}(v)}\left(n_{+}(v) z+\phi_{+}\right)^{2}+i\left(n_{+}(v)-n_{+}(b)\right) \frac{z}{2}+o(1)}
$$

as $z \rightarrow+i \infty$. Here,

$$
\phi_{+}=i \ln v_{+}-\frac{h}{2} n_{+}(b)
$$

These two solutions are linearly independent over the ring $\mathbb{K}_{+}$,

$$
\operatorname{det}\left(f_{1}(z), f_{2}(z)\right)=\frac{v_{+}}{b_{+}} .
$$

Theorem 1.1b. Let $b \not \equiv 0$, and let $v \rightarrow \infty$ as $z \rightarrow-i \infty$. There exist two Bloch solutions of (1) analytic in a vicinity $\mathbb{C}_{-}$of $-i \infty$ with the first components admitting the representations

$$
\left(g_{1,2}\right)_{1}(z)=e^{ \pm \frac{i}{2 h n_{-}(v)}\left(n_{-}(v) z+\phi_{-}\right)^{2}-i\left(n_{-}(v)-n_{-}(b)\right) \frac{z}{2}+o(1)}
$$

as $z \rightarrow-i \infty$. Here,

$$
\phi_{-}=-i \ln v_{-}-\frac{h}{2} n_{-}(b)
$$

These two solutions are linearly independent over the ring $\mathbb{K}$,

$$
\operatorname{det}\left(g_{1}(z), g_{2}(z)\right)=-\frac{v_{-}}{b_{-}}
$$

Remark 1. Note that the numbers $\phi_{ \pm}$are defined only modulo $2 \pi$. Fixing, for example, the parameter $\phi_{+}$in two different ways, we obtain two different pairs $f_{1,2}$.

Remark 2. The asymptotic formulas for $f_{1,2}$ and $g_{ \pm}$immediately imply the asymptotics of the corresponding Bloch multipliers. For $f_{1,2}$ the Bloch multipliers admit the representations

$$
\alpha_{1,2}(z)=\alpha_{1,2}^{0} e^{ \pm 2 \pi n_{+}(v) i z / h}(1+o(1)), \quad z \rightarrow+i \infty
$$


and, for $g_{1,2}$, the Bloch multipliers have the form

$$
\beta_{1,2}(z)=\beta_{1,2}^{0} e^{ \pm 2 \pi n_{-}(v) i z / h}(1+o(1)), \quad z \rightarrow+i \infty,
$$

here $\alpha_{1,2}^{0}$ and $\beta_{1,2}^{0}$ are nonzero constants,

$$
\begin{aligned}
& \alpha_{1,2}^{0}=\exp \left( \pm \frac{2 \pi i}{h}\left(\phi_{+}+\pi n_{+}(v)\right)+i \pi\left(n_{+}(v)-n_{+}(b)\right)\right) \\
& \beta_{1,2}^{0}=\exp \left( \pm \frac{2 \pi i}{h}\left(\phi_{-}+\pi n_{-}(v)\right)-i \pi\left(n_{-}(v)-n_{-}(b)\right)\right)
\end{aligned}
$$

We see that the pair $f_{1,2}$ is a basis of $\mathbb{M}_{+}$, and the solutions $g_{1,2}$ form a basis of M. We call these bases canonical. We call the number $\phi_{+}$the parameter of the canonical basis $f_{1,2}$ and the number $\phi_{-}$the parameter of the canonical basis $g_{1,2}$.

\subsection{Consistent canonical bases}

Let

$$
\Sigma=\{ \pm(2 \pi+h+2 h l+2 \pi m), l, m \in \mathbb{N}\} .
$$

We call two canonical bases $f_{1,2}$ and $g_{1,2}$ consistent if their parameters satisfy the condition

$$
\phi_{+}-\phi_{-} \notin \Sigma \text {. }
$$

Since the numbers $\phi_{ \pm}$are defined modulo $2 \pi$, the consistent bases always exist.

\subsection{Minimal entire solutions. Existence}

Let the assumptions of Theorems $1 \mathrm{a}-1 \mathrm{~b}$ be fulfilled. Fix two canonical bases $f_{1,2}$ and $g_{1,2}$ analytic in some vicinities $\mathbb{C}_{ \pm}$of $\pm i \infty$. Any entire solution of (1) admits the representations

$$
\begin{aligned}
& \psi(z)=A(z) f_{1}(z)+B(z) f_{2}(z), \quad z \in \mathbb{C}_{+}, \quad A, B \in \mathbb{K}_{+}, \\
& \psi(z)=C(z) g_{1}(z)+D(z) g_{2}(z), \quad z \in C_{-}, \quad C, D \in \mathbb{K} .
\end{aligned}
$$

Note that the periodic coefficients in these representations are uniquely defined.

We call a nonzero entire solution of (1) minimal, if

( i) its coefficients $A, B$ are bounded in $\mathbb{C}_{+}$;

( ii) the coefficients $C$ and $D$ are bounded in $\mathbb{C}_{-}$; 
(iii) at least, one of these four coefficients tends to zero as $\operatorname{Im} z$ tends to the corresponding infinity.

Note that this definition depends on the choice of the canonical bases. For a given pair of canonical bases, the definition singles out at least four minimal solutions.

One of the main results of the paper is

Theorem 1.2. Let $b(z) \not \equiv 0$, and

$$
n_{+}(v)=n_{-}(v)=n>0 .
$$

Then, for any two consistent canonical bases, there exist all the four corresponding minimal entire solutions.

Note that under the assumptions of this theorem, there exist all the canonical bases. We have already explained the condition on the coefficient $b$. Discuss the assumption (21). We shall see that among the equations (1), there is a nontrivial one which can be solved in terms of certain contour integrals. This is the equation with

$$
a(z)=-2 \lambda \cos (n z), \quad b(z)=-1, \quad c(z)=1, \quad d(z)=0,
$$

where $n \in \mathbb{N}$, and $\lambda$ is a complex number. The proof of the existence theorem is based on this observation. In the case of $(22), v(z)=2 \lambda \cos (n z)$. This leads to (21).

Now, we shall discuss the basic properties of the minimal solutions.

\subsection{Asymptotic coefficients of the minimal solutions}

Consider the representations (19) for a minimal solution. The coefficients $A, B, C$ and $D$ can be represented by the converging Fourier series

$$
\begin{gathered}
A(z)=\sum_{l=0}^{\infty} A_{l} e^{2 \pi i l z / h}, \quad B(z)=\sum_{l=0}^{\infty} B_{l} e^{2 \pi i l z / h}, \quad z \in \mathbb{C}_{+}, \\
C(z)=\sum_{l=0}^{\infty} C_{l} e^{-2 \pi i l z / h}, \quad D(z)=\sum_{l=0}^{\infty} D_{l} e^{-2 \pi i l z / h}, \quad z \in \mathbb{C}_{-} .
\end{gathered}
$$

One of the coefficients $A_{0}, B_{0}, C_{0}$ and $D_{0}$ is zero.

Assume that the Fourier coefficient $A_{0}$ equals to 0 . In this case, we denote the minimal solution by $\psi_{A}$, and call the Fourier coefficients

$$
A_{1}, \quad B_{0}, \quad C_{0} \text { and } D_{0}
$$

the asymptotics coefficients of this minimal solution. 
If $A_{0} \neq 0$, but $B_{0}=0$, we denote the minimal solution by $\psi_{B}$, and call the Fourier coefficients

$$
A_{0}, \quad B_{1}, \quad C_{0} \text { and } D_{0}
$$

the asymptotics coefficients.

Continuing in the same way, we define the minimal solutions $\psi_{C}$ and $\psi_{D}$, and their asymptotic coefficients.

In the sequel, we denote the asymptotic coefficients of the minimal solution $\psi_{A}$ by $A_{A}, B_{A}, C_{A}, D_{A}$, the asymptotic coefficients of the minimal solution $\psi_{B}$ by $A_{B}$, $B_{B}, C_{B}, D_{B}$ and so on.

The asymptotic coefficients play a crucial part in the analysis of the minimal entire solutions. Let us describe some elementary observations.

\subsection{Wronskians of the minimal solutions}

Let there exist two canonical bases $f_{1,2}$ and $g_{1,2}$ in some vicinities of $\pm i \infty$. Denote the wronskian of $f_{1,2}$ by $w_{f}$ and let $w_{g}$ be the wronskian of $g_{1,2}$. Assume also that there exist all the corresponding minimal solutions. In Section 6.1, we prove

Proposition 1.3. The wronskians of any two of the minimal solutions are constant. One has

$$
\begin{gathered}
\left\{\psi_{A}, \psi_{B}\right\}=-w_{f} B_{A} A_{B}, \\
\left\{\psi_{A}, \psi_{C}\right\}=-w_{f} B_{A} A_{C}=w_{g} C_{A} D_{C}, \quad\left\{\psi_{A}, \psi_{D}\right\}=-w_{f} B_{A} A_{D}=-w_{g} D_{A} C_{D}, \\
\left\{\psi_{B}, \psi_{C}\right\}=w_{f} A_{B} B_{C}=w_{g} C_{B} D_{C}, \quad\left\{\psi_{B}, \psi_{D}\right\}=w_{f} A_{B} B_{D}=-w_{g} D_{B} C_{D}, \\
\left\{\psi_{C}, \psi_{D}\right\}=-w_{g} D_{C} C_{D} .
\end{gathered}
$$

This statement immediately implies

Corollary 1.4. If all the asymptotic coefficients are nonzero, then any two of the minimal solutions form a basis of $\mathbb{M}$.

In Section 6.2, we obtain also

Corollary 1.5. (Uniqueness Theorem) If all the asymptotic coefficients are nonzero, then any of the minimal solutions is unique up to a constant factor.

The existence theorem is the central result of this paper. Remind that the matrix $M$ is a trigonometric polynomial. In the next publication, we shall study a family 
of the equations (1) parametrized by the constant coefficients of $M$. In particular, we shall prove that the minimal solutions and their asymptotic coefficients can be considered as meromorphic functions of these parameters, and that these functions are not identically zero. Thus, we shall see that the asymptotic coefficients are non zero for any typical matrix $M$.

\subsection{Monodromy matrices}

The notion of the minimal solution is the first of the main notions of this paper, and the second one is the notion of a monodromy matrix. We begin by recalling the general definition of a monodromy matrix and the description of the monodromization procedure, see [4].

Now, we do not need to assume that the matrix $M$ is a trigonometric polynomial. In fact, we have to suppose only that it belongs to $S L(2, \mathbb{C})$ and is a $2 \pi$-periodic matrix function.

1. Let $\Psi$ is a matrix solution of (1). We call this solution fundamental if $\operatorname{det} \Psi(z) \equiv$ Const $\neq 0$. Note that $\Psi(z+2 \pi)$ is a solution of (1) together with $\Psi(z)$. We define the monodromy matrix corresponding to a given fundamental solution $\Psi$ by the relation

$$
\Psi(z+2 \pi)=\Psi(z) M_{1}^{t}(z),
$$

where ${ }^{t}$ is the transposition. The function $M_{1}$ has the properties:

$$
\operatorname{det} M_{1}(z) \equiv 1, \quad M_{1}(z+h)=M_{1}(z) .
$$

Let $\psi^{(1)}$ and $\psi^{(2)}$ be two vector solutions of (1). Compose of them the matrix $\left(\psi^{(1)}, \psi^{(2)}\right)$. If its determinant is a nonzero constant, then this matrix is a fundamental solution. We define the monodromy matrix for such $\psi_{1}$ and $\psi_{2}$ as the monodromy matrix corresponding to the fundamental solution $\left(\psi^{(1)}, \psi^{(2)}\right)$.

The notion of a monodromy matrix is well known in the theory of the ordinary differential equations with periodic coefficients. For a differential equation

$$
\Psi^{\prime}(z)=M(z) \Psi(z)
$$

with a $2 \pi$-periodic matrix $M$, the monodromy matrix also defined by $\Psi(z+2 \pi)=$ $\Psi(z) M_{1}^{t}$, but, it is independent of $z$.

2. Recall the description of the monodromization procedure. Consider the sequence of the numbers $h_{j}, j=0,1, \ldots$, defined by the relations

$$
\begin{gathered}
h_{0}=2 \pi, \quad h_{1}=h, \\
h_{j-1}=p_{j} h_{j}+h_{j+1}, \quad 0 \leq h_{j+1}<h_{j}, \quad p_{j} \in \mathbb{N} .
\end{gathered}
$$


The $p_{j}$ are the denominators of the continued fraction

$$
h_{1} / h_{0}=\frac{1}{p_{1}+\frac{1}{p_{2}+\frac{1}{p_{3}+\ldots}}}
$$

Let $\Psi_{0}$ be a fundamental solution of equation (1), and let $M_{1}$ be the corresponding monodromy matrix. Bring into the consideration the equation

$$
\Psi_{1}\left(z+h_{2}\right)=M_{1}(z) \Psi_{1}(z) .
$$

In view of (23), this equation is of the same type as (1).

The passage from (1) to (24) is the first step of the monodromization procedure. If there exists a fundamental solution of (24), the monodromization procedure can be continued. In result, one obtains the suite of the monodromy matrices satisfying the relations:

$$
\operatorname{det} M_{j}(z) \equiv 1, \quad M_{j}\left(z+h_{j}\right)=M_{j}(z) .
$$

If the number $h_{1} / h_{0}$ is rational, the procedure is finite, In general case, the monodromization procedure is infinite.

The spectral analysis of a differential equation with periodic coefficients reduces to the study the spectrum of a (constant) monodromy matrix. Trying develop similar ideas for the difference equations, arrives to the infinite sequence of finite difference equations (23). In [3]-[4], we have seen that spectral properties of (1) with respect to the parameters of $M$ are tensely related to certain properties of the sequence $M_{1}, M_{2}, M_{3}$, etc.

\subsection{Family of matrices invariant with respect to the mon- odromization}

Denote by $\tau_{m, l}, m, l \in \mathbb{N}$, the set of the trigonometric polynomials of the form

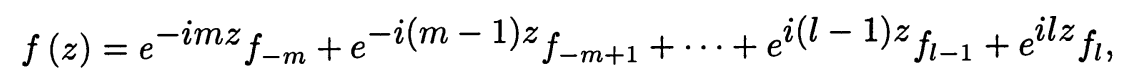

e.i. such that $n_{-}(f) \leq l, n_{+}(f) \leq m$.

Fix $n \in \mathbb{N}$. By $\Omega(n)$ or, briefly, by $\Omega$, denote the set of matrix functions $M(z) \in$ $S L(2, \mathbb{C})$ such that

$$
\begin{array}{ll}
a \in \tau_{n, n}, & b \in \tau_{n, n-1}, \\
c \in \tau_{n-1, n}, & d \in \tau_{n-1, n-1},
\end{array}
$$

and

$$
n_{+}(a)=n_{-}(a)=n .
$$


Note that the conditions (3) and (21) of the existence theorem are fulfilled for any matrix from the family $\Omega$.

One has

Theorem 1.6. Let $M \in \Omega(n)$. Consider two canonical bases $f_{1,2}$ and $g_{1,2}$. Assume that the corresponding minimal entire solutions $\psi_{D}$ and $\psi_{B}$ exist and that their wronskian is nonzero. Then the corresponding monodromy matrix as a function of the variable $z_{1}=2 \pi z / h$ also belongs to $\Omega(n)$.

This theorem can be used as the base for a program for the spectral investigation of the difference Schrödiner equations with the trigonometric polynomial potentials: the equation

$$
\frac{\psi(z+h)+\psi(z-h)}{2}+p(z) \psi(z)=E \psi(z)
$$

is equivalent to (1) with the matrix $\left(\begin{array}{cc}2 E-2 p(z) & -1 \\ 1 & 0\end{array}\right)$. In this case, $M \in \Omega(n)$, and the monodromization procedure leads to a sequence of equations (1) with the matrices from the family $\Omega(n)$. And thus, it is equivalent to a finite dimensional dynamical system. In this paper, we do not consider any spectral problems, and note only that the monodromization procedure is close to the asymptotic renormalization approach suggested for (2) by Helffer and Sjöstrand [1] and Wilkinson [10] under some semiclassical assumptions on the number $h$.

We finish this discussion by listing the asymptotic formulae for the coefficients of the monodromy matrix from Theorem 1.6:

$$
\begin{array}{lcrl}
\mathcal{M}_{11}(z) & = & \alpha_{2}^{0} e^{-2 \pi i n z / h}(1+o(1)), & z \rightarrow+i \infty,(25) \\
\mathcal{M}_{11}(z)= & \beta_{1}^{0} e^{+2 \pi i n z / h}(1+o(1)), & z \rightarrow-i \infty,(26) \\
\mathcal{M}_{12}(z)= & -\alpha_{2}^{0} e^{-2 \pi i n z / h}\left(\frac{A_{D}}{A_{B}}+o(1)\right), & z \rightarrow+i \infty,(27) \\
\mathcal{M}_{12}(z)= & -\beta_{1}^{0} e^{+2 \pi i(n-1) z / h}\left(\frac{D_{D}}{D_{B}}+o(1)\right), & z \rightarrow-i \infty,(28) \\
\mathcal{M}_{21}(z)= & \alpha_{2}^{0} e^{-2 \pi i(n-1) z / h}\left(e^{4 \pi^{2} i / h} \frac{B_{B}}{B_{D}}+o(1)\right), & z \rightarrow+i \infty,(29) \\
\mathcal{M}_{21}(z)= & \beta_{1}^{0} e^{+2 \pi i n z / h}\left(\frac{C_{B}}{C_{D}}+o(1)\right), & z \rightarrow-i \infty,(30) \\
\mathcal{M}_{22}(z)= & -\alpha_{2}^{0} e^{-2 \pi i(n-1) z / h}\left(e^{4 \pi^{2} i / h} \frac{A_{D} B_{B}}{B_{D} A_{B}}+o(1)\right), & z \rightarrow+i \infty,(31) \\
\mathcal{M}_{22}(z)= & -\beta_{1}^{0} e^{+2 \pi i(n-1) z / h}\left(\frac{D_{D} C_{B}}{D_{B} C_{D}}+o(1)\right), & z \rightarrow-i \infty .(32)
\end{array}
$$

Here $\alpha_{1,2}^{0}$ and $\beta_{1,2}^{0}$ are the constants from the asymptotic representations (16) - (17) for the Bloch multipliers of the solutions $f_{1,2}$ and $g_{1,2}$. Note also that, since the wronskian of $\psi_{D}$ and $\psi_{B}$ is nonzero, then, in view of Proposition 1.3, $A_{B}, D_{B}, B_{D}, C_{D} \neq 0$. 


\subsection{Typical properties of the minimal solutions}

Above, we have described the main results of this paper. We shall continue it in the next publication. There, we shall study some typical properties of the minimal solutions and, in particular, of their asymptotic coefficients. In fact, the second part will be devoted to the study of the dependence of the minimal solutions and their asymptotic coefficients on the parameters of the matrix $M$. Let us formulate here the central results of this second part.

Together with the set $\Omega(n)$, we consider also its subsets $\Omega_{m l},-n \leq-m \leq l \leq n-1$, singled out by the conditions

$$
n_{+}(b)=m, \quad n_{-}(b)=l .
$$

Clearly,

$$
\Omega=\bigcup_{m, l} \Omega_{m l}, \quad \text { and } \quad \Omega_{m l} \cap \Omega_{m^{\prime} l^{\prime}}=\emptyset, \quad(m, l) \neq\left(m^{\prime}, l^{\prime}\right) .
$$

It appears that the elements of $\Omega$ can be uniquely parametrized by the constant coefficients of the trigonometric polynomials $a(z)$ and $b(z)$. Denote by $\omega$ the set of constant coefficients of the matrix $M$. One can consider $\omega$ as a point in $\mathbb{C}^{8 n}$. With this, the set $\Omega_{m l},-n \leq-m \leq l \leq n-1$, appears to be a connected analytic submanifold of $\mathbb{C}^{8 n}$ of the dimension $2 n+m+l+2$, and the set $\Omega(n)$ itself is a connected analytic submanifold of $\mathbb{C}^{8 n}$ of the dimension $4 n+1$.

Let $\mathcal{D}$ be a simply connected domain of $\Omega_{m l}$. Fix some continuous on $\mathcal{D}$ branches of the functions

$$
\phi_{-}=-l h / 2-i \ln a_{+n}, \quad \phi_{+}=-m h / 2+i \ln a_{-n} .
$$

Assume that, for any point $\omega \in \mathcal{D}$, these functions satisfy the condition (18), e.i. that the canonical bases with these parameters are consistent for all $\omega \in \mathcal{D}$. One has

Theorem 1.7. For the above set $\mathcal{D}$ and the above canonical bases, the minimal entire solutions can be normalized so that they would be meromorphic in $\omega \in \mathcal{D}$ together with their asymptotic coefficients. In this case, the asymptotic coefficients are not identically zero.

This theorem and formulae (27) - (28) immediately imply

Corollary 1.8. In the case of Theorem 1.7, the monodromy matrix corresponding to the minimal solutions $\psi_{D}$ and $\psi_{B}$ is meromorphic in $\omega \in \mathcal{D}$. Moreover, this matrix typically belongs to $\Omega_{n, n-1}$. 
Here, we have used the usual terminology of the analytic set theory: one says that a property of a function defined on an analytic set is typical if it takes place outside some analytic subset of a smaller dimension.

\subsection{The plan of the paper}

In section 2, we prove Theorem 1.1. Also, we study some additional properties of the Bloch solutions.

Section 3 is devoted to the scalar equation

$$
\mu(z+h)+\mu(z-h)+2 \lambda \cos z \mu(z)=0, \quad z \in \mathbb{C},
$$

where $h>0$ and $\lambda \in \mathbb{C}$ are two fixed parameters. We construct and study minimal entire solutions of this equation. The solutions are explicitly described in terms of certain contour integrals. Note that the case of $\lambda=-1$ was already treated in [8].

Section 4 is devoted to the analysis of the equation

$$
f(z+h)+f(z-h)+2 \lambda \cos z f(z)=w(z) f(z), \quad z \in \mathbb{C},
$$

where $w$ is a meromorphic function satisfying the estimates

$$
|w(z)| \leq \text { Const } e^{-(1-\epsilon)|\operatorname{Im} z|}, \quad \epsilon>0,
$$

for sufficiently big $|\operatorname{Im} z|$. In terms of the minimal solutions of (33), we invert the operator in the left-hand side of (34), and obtain an integral Fredholm equation on a contour in the complex plane. This allows to construct and investigate some meromorphic solutions of (34).

In Section 5, we reduce the matrix equation (1) to the form (34), and then prove Theorem 1.2.

In the first part of Section 6, we study basic properties of the minimal entire solutions assuming that their asymptotic coefficients are nonzero. In particular, we prove their linear independence over the field of $h$-periodic function, their uniqueness (up to independent of $z$ factors), and justify Theorem 1.6. Recall that the monodromy matrices corresponding to the minimal solutions $\psi_{D}$ and $\psi_{B}$ are trigonometric polynomials of $2 \pi z / h$. Formulae (25) - (32) allow us to calculate only some of the constant coefficients of these polynomials. In the end of the section, we describe a way to calculate all the other coefficients.

Section 7 is devoted to some remarks for the case where $M$ is a trigonometric polynomial of the first order.

\subsection{Acknowledgments}

The work was accomplished when the authors enjoyed the hospitality of the Universities of Paris XII Val de Marne and Paris Nord. The authors are very grateful 
to the professors Alain Grigis and Colette Guillopé for their aid. The work was partially supported by the grant INTAS-RFBR 96-0414. The results of the present paper were announced in [6], see also [9].

\section{Bloch solutions in a vicinity of the infinity}

In this section, we construct Bloch solutions $f_{1,2}$ of equation (1) described in Theorem 1.1a. Subsections $2.1-2.5$ are devoted to the proof of this theorem.

In subsection 2.6 , we discuss the set of all the solutions analytic in $\mathbb{C}_{+}\left(\mathbb{C}_{-}\right)$ and having there the same asymptotic representations as $f_{1,2} \quad\left(g_{1,2}\right)$.

\subsection{The plan of the proof}

Consider equation (5) for the first component $\psi_{1}$ of a vector solution $\psi$ of equation (1). Setting

$$
\Phi(z)=\psi_{1}(z+h) / \psi_{1}(z)
$$

we see that

$$
\Phi(z)+\rho(z) / \Phi(z-h)=v(z) .
$$

We call (36) a difference Ricatti equation.

To construct a Bloch solution of (1) analytic in a vicinity of $+i \infty$ we use the ideas of [4]. The first step is to prove the existence of an analytic $2 \pi$-periodic solution $\Phi$ of the difference Ricatti equation in a vicinity of $+i \infty$. Then we introduce the function

$$
\phi(z)=\ln \Phi(z)
$$

and consider the equation

$$
\lambda(z+h)-\lambda(z)=\phi(z)
$$

Having solved this equation we reconstruct the first component of a vector solution of (1) by the formula

$$
\psi_{1}(z)=e^{\lambda(z)}
$$

after that the second one can be recovered by (6). Finally, we check that the $2 \pi$-periodicity of $\Phi$ implies that the constructed solution is a Bloch solution. 


\subsection{Analytic solutions of the Ricatti equation}

Proposition 2.1. In the case of Theorem 1.1a, there exist two $2 \pi$-periodic solutions $\Phi_{1,2}$ of (36) analytic in a vicinity of $+i \infty$ and having the asymptotics:

$$
\Phi_{2}=v(z)+o(1), \quad z \rightarrow+i \infty
$$

and

$$
\Phi_{1}(z)=\frac{\rho(z+h)}{v(z+h)}(1+o(1)), \quad z \rightarrow+i \infty
$$

Proof. Consider the sequence of the functions

$$
\begin{gathered}
\Phi^{(0)}(z)=v(z), \quad \Phi^{(1)}(z)=v(z)-\frac{\rho(z)}{v(z-h)}, \\
\Phi^{(n)}(z)=v(z)-\frac{\rho(z)}{\Phi^{(n-1)}(z-h)}, \quad n \geq 1 .
\end{gathered}
$$

The limit of this sequence (if exists) is a solution of (36) represented by the continuous fraction

$$
\Phi_{2}(z)=v(z)-\frac{\rho(z)}{v(z-h)-\frac{\rho(z-h)}{v(z-2 h)-\frac{\rho(z-2 h)}{\cdots}}} .
$$

This continuous fraction was investigated in [4].

Recall that the coefficients of the matrix $M$ are trigonometric polynomials, and that $b \not \equiv 0$. Both the functions $v$ and $\rho$ are analytic in a vicinity of $+i \infty$. Moreover, for $z \rightarrow+i \infty$, the function $\rho$ tends to a finite limit, and $v(z) \rightarrow \infty$. Let

$$
v_{-}(Y)=\inf _{\operatorname{Im} z>Y}|v(z)|, \quad \rho_{+}(Y)=\sup _{\operatorname{Im} z>Y}|\rho(z)|, \quad \mu=\frac{\rho_{+}}{\left(v_{-} / 2\right)^{2}} .
$$

As in [4], one can easily check that if $\mu<1$, then

$$
\left|\Phi^{(n+1)}(z)-\Phi^{(n)}(z)\right| \leq \mu\left|\Phi^{(n)}(z)-\Phi^{(n-1)}(z)\right|, \quad \operatorname{Im} z>Y .
$$

This estimate implies that all the functions $\Phi^{(n)}, n=1,2, \ldots$, are analytic near the "point" $+i \infty$, that the continuous fraction in (42) converges uniformly in $z$ in the half-plane $\mathbb{C}_{+}(Y)$ for sufficiently big $Y$, and that $\Phi_{2}$ satisfies the estimate (40). Since any of the functions $\Phi^{(n)}$ is $2 \pi$-periodic, the limit is also $2 \pi$-periodic.

The solution $\Phi_{1}$ can be represented by the continuous fraction

$$
\Phi_{1}(z)=\frac{\rho(z+h)}{v(z+h)-\frac{\rho(z+2 h)}{v(z+2 h)-\frac{\rho(z+3 h)}{\cdots}}} .
$$

It can be investigated in the same way as (42). q.e.d. 


\subsection{Logarithms of the solutions of the Ricatti equation}

Let

$$
\phi_{1,2}(z)=\ln \Phi_{1,2}(z)
$$

where $\Phi_{1,2}$ are the solutions of the Ricatti equation constructed in Proposition 2.1. Asymptotic representations (40) - (41) immediately imply

Lemma 2.2. In a vicinity of $+i \infty$, the functions $\phi_{1,2}(z)$ are analytic and can be represented in the form

$$
\phi_{1,2}(z)= \pm i n_{+}(v) z+\alpha_{1,2}+g_{1,2}(z)
$$

where

$$
\alpha_{2}=\ln v_{+}, \quad \alpha_{1}=-\ln v_{+}-i n_{+}(b) h+i n_{+}(v) h,
$$

and $g_{1,2}$ are $2 \pi$-periodic analytic functions decaying as $z \rightarrow+i \infty$.

\section{$2.4 \quad$ Homological equation}

Here, we collect some facts on the homological equation

$$
\varphi(z+h)-\varphi(z)=g(z), \quad z \in \mathbb{C}
$$

where $g$ is a $2 \pi$-periodic function.

1. First, prove

Lemma 2.3. Let $g$ be a $2 \pi$-periodic function analytic in a vicinity of $+i \infty$ decaying as $z \rightarrow+i \infty$, then equation (46) has a solution analytic in a vicinity of $+i \infty$ and decaying as $\operatorname{Im} z \rightarrow+\infty$ uniformly in $\operatorname{Re} z$ if $|\operatorname{Re} z|$ is bounded by a constant.

Proof. The solution $\varphi$ can be constructed by explicit formulas. Fix a point $z_{o}$ in the domain of analyticity of the function $g$. Let

$$
G(k)=\frac{1}{2 \pi} \int_{z_{\circ}}^{z_{\circ}+2 \pi} e^{-i k z} g(z) d z
$$

and

$$
\varphi(z)=\frac{1}{2 i} \int_{\gamma} G(k) e^{i k z} \frac{\operatorname{ctg} \pi k}{e^{i k h}-1} d k
$$

Here $\gamma$ is a contour in the complex plane of $k$. Describe it. 
The integrand in (47) has poles only at the real line. One of the poles is at $k=0$. One is situated at the point $k_{o}=\min \{1,2 \pi / h\}$. All the other are located outside the interval $\left[0, k_{0}\right]$. Fix two constants $c_{1}$ and $c_{2}$ so that $c_{1}>0,0<c_{2}<k_{o}$. The contour $\gamma$ is a contour coming from $+\infty$ along the line $\operatorname{Im} z=c_{1}$, going from the upper half-plane of the complex plane along the line $\operatorname{Re} z=c_{2}$, and coming back to $+\infty$ along the line $\operatorname{Im} z=-c_{1}$.

If $\operatorname{Im} z>\operatorname{Im} z_{o}$, the integral for $\varphi$ converges absolutely. Moreover,

$$
|\varphi(z)| \leq \text { Const } e^{-c_{2} \operatorname{Im} z} \text {. }
$$

This estimate is uniform in $\mathrm{Re} z$ if it is bounded by a fixed constant.

One checks that $\varphi$ satisfies equation (46) by means of the residue theorem:

$$
\begin{gathered}
\varphi(z+h)-\varphi(z)=\frac{1}{2 i} \int_{\gamma} G(k) e^{i k z} \operatorname{ctg}(\pi k) d k=\sum_{\kappa=1}^{\infty} e^{i \kappa z} G(\kappa)=g(z) . \\
\text { q.e.d. }
\end{gathered}
$$

2. Note that if $\varphi$ is a solution of (46), then the function $\tilde{g}(z)=\varphi(z+2 \pi)-\varphi(z)$ is $h$-periodic:

$$
\begin{aligned}
\tilde{g}(z+h)-\tilde{g}(z) & =\varphi(z+2 \pi+h)-\varphi(z+h)-\varphi(z+2 \pi)+\varphi(z)= \\
& =g(z+2 \pi)-g(z)= \\
& =0 .
\end{aligned}
$$

\subsection{Bloch solutions in a vicinity of $+i \infty$}

Now, we have all the ingredients to construct two Bloch solutions of (1) analytic in a vicinity of the point $+i \infty$.

\subsubsection{First solution}

Let $\Phi_{2}$ be the solution of the Ricatti equation (36) described by Proposition 2.1 and let $\phi_{2}$ be the logarithm of $\Phi_{2}$. Consider the equation

$$
\lambda(z+h)-\lambda(z)=\phi_{2}(z) .
$$

Remind that $\phi_{2}$ admits the representation (44). Therefore, any solution of (48) can be represented in the form

$$
\lambda(z)=-i n_{+}(v) \frac{z^{2}}{2 h}+i n_{+}(v) \frac{z}{2}+\ln v_{+} \frac{z}{h}+\varphi(z),
$$

where $\varphi$ is a solution of the homological equation with $g_{2}(z)$ in the right-hand side. We construct a solution $\varphi$ of this equation as in Lemma 2.3, and then, we construct 
a solution $\psi(z)$ of (1) by formulae (39) and (6). The result can be written in the form

$$
\psi(z)=e^{\lambda(z)}\left(\begin{array}{c}
1 \\
\frac{\Phi_{2}(z)-a(z)}{b(z)}
\end{array}\right) .
$$

Note that since $b(z)$ is a trigonometric polynomial, the function $\frac{1}{b(z)}$ is analytic in a vicinity of $+i \infty$, and, therefore, $\psi$ is also analytic in some vicinity $\mathbb{C}_{+}$of $+i \infty$.

Check that $\psi$ is a Bloch solution. Since the vector in the right hand side of $(50)$ is $2 \pi$-periodic,

$$
\psi(z+2 \pi)=\gamma(z) \psi(z), \quad z \in \mathbb{C}_{+}
$$

where

$$
\gamma(z)=e^{\lambda(z+2 \pi)-\lambda(z)}
$$

Thus, it suffices to show that

$$
\gamma(z+h)=\gamma(z)
$$

By (49),

$$
\gamma(z)=\text { Const } e^{-n_{+}(v) \frac{2 \pi i z}{h}} e^{\varphi(z+2 \pi)-\varphi(z) .}
$$

But, in view of section 2.3.2, the function $\varphi(z+2 \pi)-\varphi(z)$ is $h$-periodic. This implies the $h$-periodicity of $\gamma$.

Denote the constructed solution by $f_{2}$. We have come to

Lemma 2.4. There exists a Bloch solution $f_{2}$ of (1) analytic in a vicinity of $+i \infty$ with the first component admitting the representation:

$$
\left(f_{2}\right)_{1}(z)=e^{-i n_{+}(v) \frac{z^{2}}{2 h}+\ln v_{+} \frac{z}{h}+i n_{+}(v) \frac{z}{2}+o(1)}, \quad \operatorname{Im} z \rightarrow+\infty .
$$

Note that, here, $o(1)$ is just the function $\varphi$ decaying as $\operatorname{Im} z \rightarrow+i \infty$.

\subsubsection{Second solution}

Starting with $\Phi_{1}$ and repeating the above arguments we obtain

Lemma 2.5. There exists a Bloch solution $f_{1}$ of (1) analytic in a vicinity of $+i \infty$ with the first component admitting the representation:

$$
\left(f_{1}\right)_{1}(z)=e^{i n_{+}(v) \frac{z^{2}}{2 h}-\ln v_{+} \frac{z}{h}+i n_{+}(v) z / 2-i n_{+}(b) z+o(1)}, \quad \operatorname{Im} z \rightarrow+\infty .
$$




\subsubsection{Linear independence}

Calculate the determinant of the matrix $F(z) \equiv\left(f_{1}(z), f_{2}(z)\right)$. Take advantage of formula (8). This gives

$$
\operatorname{det} F(z)=\frac{\left(f_{1}\right)_{1}(z)\left(f_{2}\right)_{1}(z)}{b(z)}\left(\Phi_{2}(z)-\Phi_{1}(z)\right) .
$$

Substituting in this formula the asymptotic representations for $f_{1,2}$ and $\Phi_{1,2}$, we come to the representation

$$
\operatorname{det} F(z)=v_{+} / b_{+}(1+g(z)), \quad g(z) \rightarrow 0, \quad z \rightarrow+i \infty .
$$

Here $g$ is an $h$-periodic function, analytic in a vicinity of $+i \infty$. Note that formula (52) implies that $f_{1}$ and $f_{2}$ are linearly independent over the field of $h$-periodic functions in a vicinity of $+i \infty$.

Now, we redefine the Bloch solutions $f_{1}$ and $f_{2}$ :

$$
f_{1}:=q f_{1} /(1+g), \quad f_{2}:=f_{2} / q, \quad q=\exp \left(i \phi_{+}^{2} /\left(2 h n_{+}(v)\right)\right) .
$$

The new $f_{1}$ and $f_{2}$ are also Bloch solutions of equation (1), but their wronskian is constant. They are the solutions described in Theorem 1.1a. The proof of Theorem $1.1 b$ is absolutely similar. q.e.d.

\subsection{Uniqueness of the Bloch solutions}

1. First, we answer the question: "Are the Bloch solutions described in Theorem 1.1 uniquely defined by their asymptotics for $z \rightarrow \pm i \infty$ ?"

Lemma 2.6. Let $\tilde{f}$ be a Bloch solution of (1) analytic in some vicinity $\mathbb{C}_{+}$of $+i \infty$ and, as the solution $f_{1}$, having the asymptotics (10) for $z \rightarrow+i \infty$. Then

$$
\tilde{f}(z)=c(z) f_{1}(z)
$$

where $c(z)$ is an h-periodic function analytic in a vicinity of $+i \infty$, and having the asymptotics

$$
c(z) \rightarrow 1, \quad z \rightarrow+i \infty
$$

Proof. Since the solutions $f_{1,2}$ are linearly independent in some vicinity of $+i \infty$, $\tilde{f}$ can be represented by their linear combination with some $h$-periodic analytic coefficients

$$
\tilde{f}(z)=\alpha(z) f_{1}(z)+\beta(z) f_{2}(z) .
$$


The coefficients $\alpha$ and $\beta$ can be expressed explicitly in terms of the solutions:

$$
\alpha=\frac{\operatorname{det}\left(\tilde{f}, f_{2}\right)}{\operatorname{det}\left(f_{1}, f_{2}\right)}, \quad \beta=\frac{\operatorname{det}\left(f_{1}, \tilde{f}\right)}{\operatorname{det}\left(f_{1}, f_{2}\right)} .
$$

Since both $f_{1}$ and $\tilde{f}$ are described by (10) as $z \rightarrow+i \infty$,

$$
\alpha(z) \rightarrow 1, \quad z \rightarrow+i \infty
$$

Now, show that $\beta \equiv 0$. Since $\tilde{f}$ and $f_{1}$ are Bloch solutions

$$
\operatorname{det}\left(f_{1}, \tilde{f}\right)(z+2 \pi N)=\operatorname{det}\left(f_{1}, \tilde{f}\right)(z) \prod_{l=0}^{N-1} u_{1}(z+2 \pi l) \tilde{u}(z+2 \pi l), \quad \forall N \in \mathbb{N},
$$

where $u_{1}$ and $\tilde{u}$ are the Bloch coefficients of $f_{1}$ and $\tilde{f}$. The asymptotics (10) (see also Remark 2 to Theorems1.1) imply that, for sufficiently big $\operatorname{Im} z$,

$$
\left|u_{1}(z)\right|,|\tilde{u}(z)| \leq C e^{-2 \pi n_{+}(v) \operatorname{Im} z / h} .
$$

This formula is uniform in $\operatorname{Re} z$ since (10) is uniform in $\operatorname{Re} z$ if $|\operatorname{Re} z|$ is bounded by a constant, and since $u_{1}$ and $\tilde{u}$ are $h$-periodic. In result, for sufficiently $\operatorname{big} \operatorname{Im} z$,

$$
\left|\operatorname{det}\left(f_{1}, \tilde{f}\right)(z+2 \pi N)\right| \leq C e^{-4 \pi N n_{+}(v) \operatorname{Im} z / h}\left|\operatorname{det}\left(f_{1}, \tilde{f}\right)(z)\right|, \quad \forall N \in \mathbb{N},
$$

uniformly in Re $z$. Since the determinant is $h$-periodic, this is possible only if it equals to 0 . Thus, $\tilde{f}(z)=\alpha(z) f_{1}(z)$ which proves the lemma. $\quad$ q.e.d.

We have investigated the uniqueness of the solution $f_{1}$. For the solutions $f_{2}, g_{1}$ and $g_{2}$ one can prove the similar statements.

2. In constructing the Bloch solutions, we have fixed the parameters $\phi_{ \pm}$defined by formulae (11) and (14) only modulo $2 \pi$. The choice of the parameter $\phi_{+}\left(\phi_{-}\right)$ influences the asymptotics of $f_{1,2} \quad\left(g_{1,2}\right)$ as $z \rightarrow+i \infty \quad(z \rightarrow-i \infty)$. Fixing this parameter in two different ways, we obtain two different bases $f_{1,2}\left(g_{1,2}\right)$. Let us study relations between these bases. We shall indicate explicitly the dependence of $f_{1,2}$ on $\phi_{+}$. On has

\section{Lemma 2.7.}

$$
f_{1,2}\left(z, \phi_{+}+2 \pi\right)=c_{1,2} e^{ \pm 2 \pi i z / h} f_{1,2}\left(z, \phi_{+}\right)
$$

where $c_{1,2}=c_{1,2}\left(z, \phi_{+}\right)$are some analytic $h$-periodic functions tending to nonzero constants as $z \rightarrow+i \infty$. 
The proof of this lemma is similar to the proof of the previous one. Again, one can prove the same statement for the Bloch solutions $g_{1,2}$.

The most interesting case is one where we change $\phi_{+}$by $2 \pi n_{+}(v)$. In this case, one comes to

\section{Corollary 2.8.}

$$
f_{1,2}\left(z, \phi_{+}+2 \pi n_{+}(v)\right)=C_{1,2} f_{1,2}\left(z+2 \pi, \phi_{+}\right),
$$

where $C_{1,2}=C_{1,2}\left(z, \phi_{+}\right)$are some analytic $h$-periodic functions with the asymptotics

$$
C_{1,2}\left(z, \phi_{+}\right)=e^{-\pi i\left(n_{+}(v)-n_{+}(b)\right)}+o(1), \quad z \rightarrow+i \infty .
$$

\section{Minimal solution of the auxiliary equation}

The next three sections are devoted to constructing of the minimal solutions of equation (1). Let us agree on the terminology and the notations we shall use there.

For $z \in \mathbb{C}$, we call the set

$$
\{\zeta \in \mathbb{C}: \operatorname{Im} z=\operatorname{Im} \zeta,-\delta<\operatorname{Re}(z-\zeta)<\delta\}
$$

the horizontal $\delta$-vicinity of $z$. We call the horizontal $\delta$-vicinity of a curve $\gamma \subset \mathbb{C}$ the union of the horizontal $\delta$-vicinities of the all its points. We call the horizontal distance between a curve $\gamma$ and a point $z$ the maximal $\delta$ for which the horizontal $\delta$-vicinity of $\gamma$ does not contain $z$.

We call a curve $\gamma \subset \mathbb{C}$ vertical, if it intersects all the lines $\operatorname{Im} z=$ Const only at nonzero angles. If all these angles are strictly bigger than some fixed positive constant, we call the curve strictly vertical.

Furthermore, in the sequel, we use the letter $C$ as a symbol denoting constants independent of $z$.

In Section 3, we construct and investigate minimal entire solutions of the model equation

$$
\left(\begin{array}{l}
m_{1}(z+h) \\
m_{2}(z+h)
\end{array}\right)=\left(\begin{array}{cc}
-2 e^{\xi} \cos z & -1 \\
1 & 0
\end{array}\right)\left(\begin{array}{l}
m_{1}(z) \\
m_{2}(z)
\end{array}\right), \quad z \in \mathbb{C}
$$

where $\xi$ is a complex number. Note that the first component $m_{1}$ of its vector solutions satisfies the equation

$$
m(z+h)+m(z-h)+2 e^{\xi} \cos z m(z)=0,
$$


and the second component is related to $m_{1}$ by the formula $m_{2}(z)=m_{1}(z-h)$. The main results are formulated in Theorem 3.2. When describing the results, we use the special function described in Section 3.2.

In [8], we have considered the case where $\xi=0$. Here, we discuss in details only the new elements of the proof.

\subsection{Reduction to a first order difference equation}

We shall construct a solution $m(z)$ of $(56)$ in the form:

$$
m(z)=e^{-i z^{2} / 2 h} \int_{\Gamma} e^{-i z p / h-i p^{2} / 4 h+\pi i p / 2 h} v(p) d p
$$

where $\Gamma$ is a contour in the complex plane of $p$. Substituting (57) into (56), we formally get

$$
\begin{aligned}
& e^{-i z} \int_{\Gamma} e^{-i z p / h-i p^{2} / 4 h+\pi i p / 2 h}\left(e^{-i p-i h / 2}+e^{\xi}\right) v(p) d p+ \\
& \quad e^{+i z} \int_{\Gamma} e^{-i z p / h-i p^{2} / 4 h+\pi i p / 2 h}\left(e^{i p-i h / 2}+e^{\xi}\right) v(p) d p=0
\end{aligned}
$$

or, after having changed the variables,

$$
\begin{gathered}
\int_{\Gamma+h} e^{-i z p / h-i(p-h)^{2} / 4 h+\pi i(p-h) / 2 h}\left(e^{-i p+i h / 2}+e^{\xi}\right) v(p-h) d p+ \\
\int_{\Gamma-h} e^{-i z p / h-i(p+h)^{2} / 4 h+\pi i(p+h) / 2 h}\left(e^{i p+i h / 2}+e^{\xi}\right) v(p+h) d p=0
\end{gathered}
$$

where $\Gamma \pm h$ are the contours obtained of $\Gamma$ by $\pm h$-translations. Assuming that $v$ is analytic in a sufficiently large vicinity of $\Gamma$, and that there are no problems of convergence of the integrals, we deform the integration contours back to $\Gamma$ and obtain

$$
\begin{gathered}
\int_{\Gamma} e^{-i z p / h-i p^{2} / 4 h+\pi i p / 2 h}\left[\left(e^{-i p / 2+i h / 2}+e^{\xi+i p / 2}\right) v(p-h)\right. \\
\left.-\left(e^{i p / 2+i h / 2}+e^{\xi-i p / 2}\right) v(p+h)\right] d p=0 .
\end{gathered}
$$

So, if $v$ satisfies the first order difference equation

$$
v(p+h)=\varrho(p) v(p-h)
$$

with

$$
\varrho(p)=\frac{e^{i h / 2}+e^{i p+\xi}}{e^{\xi}+e^{i h / 2+i p}},
$$

then $m$ is a solution of (56). Now, our aim is to construct a solution of (58). To get a detailed information about this solution we shall need a special function playing an important role in the analytic theory of difference equations with periodic coefficients. 


\section{$3.2 \quad \sigma$-function}

Here, we construct a meromorphic solution of the equation

$$
\sigma(z+h)=\left(1+e^{-i z}\right) \sigma(z-h), \quad z \in \mathbb{C} .
$$

A similar function was used also in the diffraction theory [2] , so we carry out the construction omitting elementary details.

1. Let $\mathbb{C}_{\pi}$ be the complex plane cut along the real line from $-\infty$ to $-\pi$, and from $\pi$ to $+\infty$. Fix on $\mathbb{C}_{\pi}$ a branch of the function $l_{0}(z)=\ln \left(1+e^{-i z}\right)$ by the condition

$$
l_{0}(z) \rightarrow 0, \quad z \rightarrow-i \infty \text {. }
$$

Define

$$
L_{0}(z)=\int_{-i \infty}^{z} l_{0}\left(z^{\prime}\right) d z^{\prime}, \quad z \in \mathbb{C}_{\pi},
$$

where the integration contour belongs to $\mathbb{C}_{\pi}$. Let

$$
\theta_{0}(z)=\frac{\pi}{8 i h^{2}} \int_{\gamma} \frac{L_{0}\left(z^{\prime}\right)}{\cos ^{2}\left(\frac{\pi\left(z-z^{\prime}\right)}{2 h}\right)} d z^{\prime} .
$$

Here, $\gamma \subset \mathbb{C}_{\pi}$ is a strait line $\operatorname{Re} z=$ Const passing between the points $z \pm h$. The function $\theta_{0}$ is analytic in the strip $S_{0}=\{z \in \mathbb{C}:-\pi-h<\operatorname{Re} z<\pi+h\}$.

By means of the residue theorem, one can easily check that

$$
\theta_{0}(z+h)-\theta_{0}(z-h)=l_{0}(z), \quad z \pm h \in S_{0} .
$$

Therefore, the function

$$
\sigma(z)=e^{\theta_{0}(z)}
$$

is a solution of (59) in the strip $S_{0}$. By means of (59), one can continue it meromorphically in the whole complex plane. Let now $\sigma$ denote this meromorphic solution. Investigate its analytic properties.

2. Discuss the set of poles and zeros of the function $\sigma$. By construction, the function $\sigma$ is analytic and has no zeros in $S_{0}$. In result of the continuation, each zero $z=\pi+2 \pi l, l=0,1,2, \ldots$, of the function $1+e^{-i z}$ produces a chain of zeros of $\sigma$ at the points

$$
\pi+h+2 \pi l+2 h k, \quad k=0,1,2, \ldots,
$$

and each its zero $z=-\pi-2 \pi l, l=0,1,2, \ldots$, produces a chain of poles of $\sigma$ at the points

$$
-\pi-h-2 \pi l-2 h k, \quad k=0,1,2, \ldots
$$

3. The pole $z=-\pi-h$ is simple. One can calculate explicitly the residue of $\sigma$. Omitting long elementary calculations, we write down the result

$$
\operatorname{Res}_{z=-\pi-h} \sigma=-i \sigma(-\pi+h)=\sqrt{\frac{h}{\pi}} e^{-\frac{i \pi^{2}}{12 h}-\frac{i \pi}{4}-\frac{i h}{12}} .
$$


One can also get the following explicit formula

$$
\sigma(-\pi)=\frac{1}{\sqrt{2}} e^{-i \pi^{2} / 12 h+i h / 24}
$$

4. Let us describe the asymptotics of $\sigma$ for $\operatorname{Im} z \rightarrow \pm i \infty$. Clearly,

$$
L_{0}(z)=O\left(e^{-|\eta|}\right), \quad \operatorname{Im} z \rightarrow-i \infty .
$$

On the other hand,

$$
L_{0}(z)=-i z^{2} / 2+i \pi^{2} / 6+O\left(e^{-|\eta|}\right), \quad \operatorname{Im} z \rightarrow+i \infty .
$$

This implies the asymptotics

$$
\sigma(z)=1+o\left(e^{-\mu|\operatorname{Im} z|}\right), \quad \operatorname{Im} z \rightarrow-i \infty,
$$

and

$$
\sigma(z)=e^{-i \frac{z^{2}}{4 h}+i \frac{\pi^{2}}{12 h}+i \frac{h}{12}}\left(1+o\left(e^{-\mu|\operatorname{Im} z|}\right)\right), \quad \operatorname{Im} z \rightarrow+i \infty,
$$

where $\mu$ is a positive number such that $\mu<\min \{1, \pi / h\}$. As before, we omit elementary calculations, noticing only that the leading terms in (65) can be obtained by the substitution in (61) instead of the function $L_{0}$ the leading terms of its asymptotics for $z \rightarrow+i \infty$.

5. The solution $\sigma$ of equation (59) is uniquely determined by its asymptotics (64) - (65), and by the fact that it is analytic and has no zeros in the strip $S_{0}$.

Indeed, let $\sigma_{1}$ be another solution of (59) possessing these properties. By (59), the ratio $\sigma_{1}(z) / \sigma(z)$ a $2 h$-periodic function. It is analytic in $S_{0}$, and thus, due to the periodicity, it is entire. On the other hand, it tends to 1 as $z \rightarrow \pm i \infty$. Therefore, this ratio is identically equal to 1 .

6. The $\sigma$-function satisfies the functional relations:

$$
\begin{gathered}
\sigma(z+\pi)=\left(1+e^{-\frac{i \pi}{h} z}\right) \sigma(z-\pi) \\
\sigma(-z)=e^{-\frac{i}{4 h} z^{2}+\frac{i \pi^{2}}{12}+\frac{i h}{12}} \frac{1}{\sigma(z)} \\
\overline{\sigma(\bar{z})}=\frac{1}{\sigma(-z)}
\end{gathered}
$$

These three relations can be proved by using almost one and the same argument. To justify, for example, formula (66), first, one checks that the ratio $r(z)=\sigma(z) / \sigma(z-$ 
$2 \pi)$ is a $2 h$-periodic entire function. Really, both the functions $\sigma(z)$ and $\sigma(z-2 \pi)$ satisfy (59). This implies that $r$ is $2 h$-periodic. The function $\sigma(z)$ is analytic in the half-plane $-\pi-h<\operatorname{Re} z$, and $1 / \sigma(z-2 \pi)$ is analytic in the half-plane $\operatorname{Re} z<3 \pi+h$. So, $r$ is analytic in the strip $-\pi-h<\operatorname{Re} z<3 \pi+h$. Thus, being $2 h$-periodic, it is entire. Furthermore, the asymptotics of $\sigma$ imply that $r(z) \rightarrow 1$ as $z \rightarrow-i \infty$, and $r(z)=e^{-i \pi z / h+i \pi^{2} / h}(1+o(1))$ as $z \rightarrow+i \infty$. This is possible only if $r(z)=1+e^{\frac{i \pi^{2}}{h}}-\frac{i \pi}{h} z$, which proves $(66)$.

\subsection{Meromorphic solution of equation (58)}

Now, we come back to equation (58). One of its solution can be constructed by the formula

$$
v(p)=e^{-i \frac{p_{0}}{2 h} p} \sigma\left(p-p_{0}\right) / \sigma\left(p+p_{0}\right)
$$

where

$$
p_{0}=i \xi+h / 2 \text {. }
$$

Describe analytic properties of $v$.

First of all, note that (67) implies that

$$
v(-p)=v(p) .
$$

The function $v$ has two chains of poles:

$$
\pm\left(-p_{o}+\pi+h+2 \pi j+2 h k\right), \quad j, k \in \mathbb{N} \cup\{0\} .
$$

If these two chains do not intersect, then the poles $\pm\left(-p_{0}+\pi+h\right)$ are simple, and

$$
\begin{aligned}
\operatorname{Res}_{p_{0}-\pi-h} v(p) & =-\operatorname{Res}_{-p_{0}+\pi+h} v(p)= \\
& =\sqrt{\frac{h}{\pi}} e^{i \xi^{2} / 2 h-\pi \xi / 2 h-i \pi^{2} / 12 h+i h / 24} \frac{1}{\sigma(2 i \xi-\pi)} .
\end{aligned}
$$

The asymptotic formulae for $\sigma$ imply

$$
v(p)=e^{ \pm i p_{0} p / 2 h}(1+o(1)), \quad p \rightarrow \pm i \infty .
$$

\subsection{Minimal solution of the model equation for $\operatorname{Re} p_{0}<\pi$}

In this section we assume that

$$
\operatorname{Im} \xi>-\pi+h / 2 .
$$


This condition is equivalent to the inequality $\operatorname{Re} p_{0}<\pi$. It implies that the poles of $v$ are outside the strip

$$
-h \leq \operatorname{Re} p \leq h
$$

1. Construct an entire solution of the auxiliary equation (56). Let $\Gamma$ be a vertical curve going along the imaginary axis from $p=-i \operatorname{Re} \xi$ to $p=+i \operatorname{Re} \xi$ and having as the asymptotes for $p \rightarrow \pm i \infty$ the line $e^{-i \pi / 4} \mathbb{R}$. Since $v$ has the asymptotics (72), the integral in (57) converges and defines an entire function $m(z)$.

This function $m$ is an entire solution of the auxiliary equation (56). To check this, we make the calculation from subsection 3.1. As the convergence of the integrals is obvious, then, to justify this calculation, one has only to note that the horizontal distance from the contour $\Gamma$ to the poles of $v$ is bigger than $h$.

2. Let us turn the attention to the asymptotics of $m$ for $z \rightarrow \pm i \infty$. One has

Proposition 3.1. Let $\operatorname{Im} \xi>-\pi+h / 2$. Then the function $m$ has the asymptotics

$$
\begin{aligned}
& m(z)=a_{0} e^{\frac{i}{2 h}(z-\pi+i \xi)^{2}+\frac{i z}{2}}(1+o(1))+ \\
& +b_{0} e^{-\frac{i}{2 h}(z-\pi+i \xi)^{2}+\frac{i z}{2}}(1+o(1)), \quad z \rightarrow+i \infty \\
& m(z)=c_{0} e^{\frac{i}{2 h}(z-\pi-i \xi)^{2}-\frac{i z}{2}}(1+o(1))+ \\
& +e^{-2 \pi i z / h} d_{0} e^{-\frac{i}{2 h}(z-\pi-i \xi)^{2}-\frac{i z}{2}}(1+o(1)), \quad z \rightarrow-i \infty
\end{aligned}
$$

where $a_{0}, b_{0}, c_{0}$ and $d_{0}$ are independent of $z$. The asymptotics are uniform in $\operatorname{Re} z$ if $|\operatorname{Re} z|$ is bounded by a fixed constant. The asymptotic representations for $m^{\prime}$ can be obtained by differentiating the asymptotic representations for $m$.

When proving the proposition, one checks also that the constant coefficients in the above asymptotics are given by the formulae:

$$
\begin{gathered}
a_{0}=2 i \sqrt{\pi h} e^{-\frac{i}{4 h}(i \xi-\pi)^{2}-\frac{1}{4} \xi+\frac{i h}{16}} \\
b_{0}=\frac{2 \sqrt{\pi h}}{\sigma(2 i \xi-\pi)} e^{-\frac{i}{4 h}(\pi-i \xi)^{2}-\frac{1}{4} \xi-\frac{i \pi^{2}}{12 h}-\frac{i h}{48}} \\
c_{0}=-2 \sqrt{\pi h} e^{-\frac{i}{4 h}(\pi+i \xi)^{2}-\frac{1}{4} \xi+\frac{i h}{16}}
\end{gathered}
$$




$$
d_{0}=\frac{2 \sqrt{\pi h} i}{\sigma(2 i \xi-\pi)} e^{-\frac{2 \pi \xi}{h}-\frac{i}{4 h}(\pi+i \xi)^{2}-\frac{1}{4} \xi+\frac{11 i \pi^{2}}{12 h}-\frac{i h}{48}}
$$

For the case where $\xi \equiv 0$, we have proved this proposition in [8]. In the case under the consideration, one can use the same arguments and, even, almost the same estimates and calculations. So, we shall not repeat them here, and give only some comments on the proof.

Begin with the case of $z \rightarrow+i \infty$. Then, the asymptotics contains two leading terms. The first one is defined by the behavior of $v$ as $p \rightarrow-i \infty$, and the second one is related to the pole of the function $v$ situated at $p_{0}-\pi-h$. Describe the first one.

Remind that as $p \rightarrow \pm i \infty$, the integrand in (57) has the asymptotics

$$
e^{-\frac{i z}{h} p-\frac{i}{4 h} p^{2}+\frac{i \pi}{2 h} p \pm \frac{i p_{0}}{2 h} p_{(1+o(1))} .}
$$

If $z \rightarrow+i \infty$, the integrand has a saddle point. It satisfies the equation

$$
\frac{d}{d p}\left(-\frac{i z}{h} p-\frac{i}{4 h} p^{2}+\frac{i \pi}{2 h} p-\frac{i p_{0}}{2 h} p\right)=0,
$$

and thus, the saddle point is $p_{1}=-2 z+\pi-p_{0}$. The first of the leading terms equals to the contribution of this saddle point to (57), e.i. to

$$
\begin{aligned}
e^{-\frac{i}{2 h} z^{2} \sqrt{4 \pi h} e^{\frac{3 \pi i}{4}}} e^{-\frac{i}{h} z p_{1}-\frac{i}{4 h} p_{1}^{2}+\frac{i \pi}{2 h} p_{1}-\frac{i}{2 h} p_{0} p_{1}}= & \\
= & a_{0} e^{\frac{i}{2 h}(z-\pi+i \xi)^{2}+\frac{i z}{2}}
\end{aligned}
$$

where $a_{0}$ is given by (77).

The second leading term of the asymptotics of $m(z)$ equals to the contribution of the pole $p_{0}-\pi-h$ to $(57)$, e.i. to

$$
\begin{gathered}
e^{-\frac{i}{2 h} z^{2}} 2 \pi i \operatorname{Res}_{p=p_{0}-\pi-h}\left(e^{-i z p / h-i p^{2} / 4 h+\pi i p / 2 h} v(p)\right)= \\
=b_{0} e^{-\frac{i}{2 h}(z-\pi+i \xi)^{2}+\frac{i}{2} z}
\end{gathered}
$$

with $b_{0}$ from (78).

The asymptotics (75) of $m(z)$ for $z \rightarrow+i \infty$ can be described as the sum of the contributions of the above two terms.

In the case where $z \rightarrow-i \infty$, the asymptotic (76) of $m$ again contains two leading terms. The first one is due to the saddle point $p_{2}$ defined by

$$
\frac{d}{d p}\left(-\frac{i z}{h} p-\frac{i}{4 h} p^{2}+\frac{i \pi}{2 h} p+\frac{i p_{0}}{2 h} p\right)=0,
$$


i.e. $p_{2}=-2 z+\pi+p_{0}$. The contribution of this saddle point to (57) is given by

$$
\begin{aligned}
& e^{-\frac{i}{2 h} z^{2}} \sqrt{4 \pi h} e^{\frac{3 \pi i}{4}} e^{-\frac{i}{h} z p_{2}-\frac{i}{4 h} p_{2}^{2}+\frac{i \pi}{2 h} p_{2}+\frac{i}{2 h} p_{0} p_{2}}= \\
& =c_{0} e^{\frac{i}{2 h}(z-\pi-i \xi)^{2}-\frac{i z}{2}},
\end{aligned}
$$

where $c_{0}$ is given by (79). This is the first leading term, and the second one equals to

$$
\begin{array}{r}
-e^{-\frac{i}{2 h} z^{2}} 2 \pi i \operatorname{Res}_{p=-p_{0}+\pi+h}\left(e^{-i z p / h-i p^{2} / 4 h+\pi i p / 2 h} v(p)\right)= \\
=e^{-2 \pi i z / h} d_{0} e^{-\frac{i}{2 h}(z-\pi-i \xi)^{2}-\frac{i z}{2}}
\end{array}
$$

q.e.d.

3. Recall that we can reconstruct the components of a vector solution of (55) by the formule $m_{1}(z)=m(z)$ and $m_{2}(z)=m(z-h)$. This vector solution is a minimal entire solution. Let us discuss this in detail.

The theorems $1.1 \mathrm{a}$ and $1.1 \mathrm{~b}$ imply existence of the canonical vector solutions $f_{1,2}$ and $g_{1,2}$ of (55). The asymptotics of their first components have the form

$$
\begin{gathered}
\left(f_{1,2}\right)_{1}(z)=e^{ \pm \frac{i}{2 h}\left(z+\phi_{+}\right)^{2}+i \frac{z}{2}+o(1)}, \quad z \rightarrow+i \infty \\
\phi_{+}=i \xi-\pi
\end{gathered}
$$

and

$$
\begin{gathered}
\left(g_{1,2}\right)_{1}(z)=e^{ \pm \frac{i}{2 h}\left(z+\phi_{-}\right)^{2}-i \frac{z}{2}+o(1)}, \quad z \rightarrow-i \infty \\
\phi_{-}=-i \xi-\pi
\end{gathered}
$$

Here, we have chosen some of the possible values of the parameters $\phi_{ \pm}$. Comparing the asymptotics of $m_{1}(z)=m(z)$ (described by Proposition 3.1) with (81) - (82), we see that the vector $\left(\begin{array}{c}m_{1}(z) \\ m_{2}(z)\end{array}\right)$ is really a minimal solution of (55) corresponding to the chosen canonical bases.

Below, we shall refer to $m$ as to a minimal entire solution of (56).

\subsection{Minimal solution in the general case}

In the previous section, we have constructed the minimal entire solution $m(z)$ for $\xi$ being in the half-plane (73). It is analytic in $\xi$ in this half-plane. Now, we are going to continue it meromorphically on the whole complex plane of $\xi$ and, in result, to prove

Theorem 3.2. Equation (56) has a solution $m(z, \xi)$ entire in $z$ and meromorphic in $\xi$. It has the following properties: 
(i) $m$ is analytic in $\xi$ in the upper half-plane of the complex plane $\xi$, and the function $\sigma(2 i \xi-\pi) m(z, \xi)$ is analytic in the half-plane $\operatorname{Im} \xi \leq 0$.

(ii) If $\sigma(2 i \xi-\pi) \neq 0$, then the behavior of $m$ for $z \rightarrow \pm i \infty$ is described by formulae $(75)-(76)$ with coefficients $a_{0}, b_{0}, c_{0}$ and $d_{0}$ given by $(77)-(80)$.

Above, we have indicated explicitly the dependence of $m$ on $\xi$. The rest of the section is devoted to the proof.

1. Let us study analytic properties of $m$ as a function of $\xi$. As we have already noted, it is analytic in $\xi$ if $\operatorname{Im} \xi>-\pi+h / 2$. Continue meromorphically $m(z, \xi)$ into the whole complex plane of $\xi$. The idea is to find a relation connecting the functions $m(z, \xi), m(z, \xi+i \pi)$ and $m(z, \xi+2 i \pi)$ for $\operatorname{Im} \xi>-\pi+h / 2$, and to use this relation for the continuation. Such a relation exists since all the three functions $m(z, \xi), e^{i \pi z / h} m(z, \xi+i \pi)$ and $m(z, \xi+2 i \pi)$ satisfy (56), and since the space of solutions of the equivalent to (56) equation (55) is a two dimensional module over the ring of $h$-periodic functions. Prove

Lemma 3.3. If $\operatorname{Im} \xi>-\pi+h / 2$, then

$$
\sigma(2 i \xi-\pi) m(z, \xi)=\sigma(2 i \xi-3 \pi)[\alpha m(z, \xi+i \pi)+\beta m(z, \xi+2 i \pi)],
$$

where $\alpha$ and $\beta$ are entire functions of $\xi$ and entire and h-periodic in $z$.

Proof. As equation (55) and (56) are equivalent, then the space of solutions of (56) is also a two dimensional module over the ring of $h$-periodic functions $\mathbb{K}$, and, moreover, for any two solutions $f$ and $g$ of (56), the expression

$$
\{f(z), g(z)\}=f(z+h) g(z)-f(z) g(z+h)
$$

is $h$-periodic, and $f$ and $g$ are linearly independent over $\mathbb{K}$ iff $\{f(z), g(z)\} \not \equiv 0$. We call $\{f(z), g(z)\}$ the wronskian of $f$ and $g$.

Assuming additionally to the hypothesis of the lemma that $\xi$ satisfies the condition

$$
1 / \sigma(2 i \xi-3 \pi) \neq 0
$$

e.i. that

$$
\xi \neq-i \pi+i h / 2+i \pi l+i h k, \quad l, k=0,1,2, \ldots,
$$

we check that $e^{i \pi z / h} m(z, \xi+i \pi)$ and $m(z, \xi+2 i \pi)$ form a base in the space of entire solutions of (56). For this, we calculate their wronskian. We shall write $f \sim g$ if $f$ differs from $g$ by an entire in $\xi$ factor having no zeros.

First, we study the asymptotics of the wronskian for $z \rightarrow \pm i \infty$. They can be easily calculated by means of the asymptotics of $m$. Assuming that $b_{0}(\xi+i \pi)$ and $a_{0}(\xi+2 i \pi)$ are nonzero, we get

$$
\left\{e^{i \pi z / h} m(z, \xi+i \pi), m(z, \xi+2 i \pi)\right\} \sim b_{0}(\xi+i \pi) a_{0}(\xi+2 i \pi)(1+o(1)) .
$$




$$
z \rightarrow+i \infty
$$

But, in fact, formula (77) shows that $a_{0}(\xi) \neq 0$ for all $\xi \in \mathbb{C}$, and formula (78) implies that $b_{0}(\xi+i \pi) \neq 0$ under the condition (84). This justifies (85). Similarly, one shows that

$$
\begin{gathered}
\left\{e^{i \pi z / h} m(z, \xi+i \pi), m(z, \xi+2 i \pi)\right\} \sim d_{0}(\xi+i \pi) c_{0}(\xi+2 i \pi)(1+o(1)) . \\
z \rightarrow-i \infty
\end{gathered}
$$

Since the wronskian is an entire $h$-periodic function of $z,(85)$ and (86) imply that it is in fact independent of $z$,

$$
\left\{e^{i \pi z / h} m(z, \xi+i \pi), m(z, \xi+2 i \pi)\right\} \sim d_{0}(\xi+i \pi) c_{0}(\xi+2 i \pi) .
$$

Furthermore, taking into account (79) and (80), we see that

$$
\left\{e^{i \pi z / h} m(z, \xi+i \pi), m(z, \xi+2 i \pi)\right\} \sim 1 / \sigma(2 i \xi-3 \pi),
$$

and thus, under the condition (84), $e^{i \pi z / h} m(z, \xi+i \pi)$ and $m(z, \xi+2 i \pi)$ are basis solutions. Therefore, in this case, $m(z, \xi)$ is their linear combination,

$$
m(z, \xi)=\tilde{\alpha} e^{i \pi z / h} m(z, \xi+i \pi)+\tilde{\beta} m(z, \xi+2 i \pi)
$$

with the coefficients

$$
\begin{gathered}
\tilde{\alpha}=\{m(z, \xi), m(z, \xi+2 i \pi)\} /\left\{e^{i \pi z / h} m(z, \xi+i \pi), m(z, \xi+2 i \pi)\right\}, \\
\tilde{\beta}=\left\{e^{i \pi z / h} m(z, \xi+i \pi), m(z, \xi)\right\} /\left\{e^{i \pi z / h} m(z, \xi+i \pi), m(z, \xi+2 i \pi)\right\} .
\end{gathered}
$$

One can calculate the wronskians in these formulae in the same manner as above, which gives

$$
\begin{gathered}
\{m(z, \xi), m(z, \xi+2 i \pi)\}=w_{1}(\xi)+w_{2}(\xi) e^{-\frac{2 \pi i}{h} z} \\
\left\{e^{i \pi z / h} m(z, \xi+i \pi), m(z, \xi)\right\}=w_{3}(\xi), \\
w_{j} \sim 1 / \sigma(2 i \xi-\pi), \quad j=1,2,3 .
\end{gathered}
$$

This and (87) imply the desired relation (83) under the condition (84). But, then it is valid for any $\xi$ in the half-plane $\operatorname{Im} \xi>-\pi+h / 2$ as an equality of two meromorphic functions.

q.e.d.

2. Now, we note that the integral (57) remains analytic in $\xi$ and entire in $z$ while the pole $p_{0}-\pi-h=i \xi-\pi-h / 2$ of the function $v$ remains in the left half-plane of the complex plane, and its pole $-p_{0}+\pi+h=-i \xi+\pi+h / 2$ remains in the right half-plane. Thus, $m$ is analytic in $\xi$ if $\operatorname{Im} \xi>-\pi-h / 2$.

The above result implies that Lemma 3.3 remains valid for all $\xi$ in the half-plane $\operatorname{Im} \xi>-\pi-h / 2$. 
3. Consider the function

$$
\Phi(z, \xi)=\sigma(2 i \xi-\pi) m(z, \xi) .
$$

Since the poles of $\sigma(2 i \xi-\pi)$ are in the half-plane $\operatorname{Im} \xi \geq h / 2, \Phi$ is analytic in $\xi$ in the strip $-\pi-h / 2<\operatorname{Im} \xi<h / 2$. Lemma 3.3 immediately implies

Lemma 3.4. The function $\Phi$ can be analytically continued in the half-plane $\operatorname{Im} \xi<$ $h / 2$.

Proof. The right hand side in (83) is analytic in $\xi$ if $-2 \pi-h / 2<\operatorname{Im} \xi<-\pi+h / 2$. Therefore, $\Phi$ is analytic in the strip $-2 \pi-h / 2<\operatorname{Im} \xi<h / 2$. By means of (66), one can rewrite formula (83) in the form

$$
\Phi(z, \xi)=\alpha(z, \xi) \Phi(z, \xi+i \pi)+\beta(z, \xi)\left(1+e^{\frac{2 \pi}{h}(\xi+2 i \pi)}\right) \Phi(z, \xi+2 i \pi),
$$

and this relation allows to continue $\Phi$ analytically from the strip $-2 \pi-h / 2<$ $\operatorname{Im} \xi<h / 2$ in the whole half-plane $\operatorname{Im} \xi<h / 2$.

q.e.d.

The lemma implies that $m(z, \xi)$ can be meromorphically continued on the whole complex plane. We shall not distinguish between $m$ and its meromorphic continuation. The $m$ is analytic in $\xi$ in the upper half-plane of the complex plane, and the product $\sigma(2 i \xi-\pi) m(z, \xi)$ is analytic in the half-plane $\operatorname{Im} \xi \leq 0$.

4. Remind that $m$ is entire in $z$ for $\operatorname{Im} \xi>-\pi+h / 2$. As the meromorphic continuation of $m$ into the whole plane of $\xi$ was obtained by (83), $m$ is entire in $z$ for all $\xi$ different from the zeros of $\sigma(2 i \xi-\pi)$, i.e. outside the poles of $m$.

5. Since $m(z+h, \xi), m(z, \xi)$ and $m(z-h, \xi)$ are meromorphic in $\xi$, equation (56) remains satisfied for all $\xi \in \mathbb{C}$ as a relation for meromorphic in $\xi$ functions.

6. To complete the proof of the theorem, we have to check only its statement (ii). Consider the canonical Bloch solutions of (56) described by (81) - (82). One can represent $\Phi(z, \xi)=\sigma(2 i \xi-\pi) m(z, \xi)$ by their linear combination with $h$-periodic coefficients. In a vicinity $\mathbb{C}_{+}$of $+i \infty$,

$$
\Phi(z, \xi)=A(z, \xi) f_{1}(z, \xi)+B(z, \xi) f_{2}(z, \xi), \quad z \in \mathbb{C}_{+} .
$$

The coefficients $A(., \xi), B(., \xi)$ can be expressed in terms of the wronskians of $\Phi$ and $f_{1,2}$ :

$$
A=\left\{\Phi, f_{2}\right\} /\left\{f_{1}, f_{2}\right\}, \quad B=\left\{f_{1}, \Phi\right\} /\left\{f_{1}, f_{2}\right\} .
$$

Thus, they are analytic in $\xi$ in the same part of the complex plane as $\Phi$. Also, all the Fourier coefficients of $A$ and $B$ are analytic in $\xi$ in the same domain as $\Phi$, and, therefore are described by the same analytic formulae as for $\operatorname{Im} \xi>-\pi+h / 2$, so everywhere outside the poles of $\sigma$. The asymptotics (75) - (76) show that

$$
A \rightarrow a_{0}(\xi) \sigma(2 i \xi-\pi), \quad B \rightarrow b_{0}(\xi) \sigma(2 i \xi-\pi),
$$




$$
\operatorname{Im} \xi>-\pi+h / 2, \quad z \rightarrow+i \infty
$$

outside the poles of $\sigma(2 i \xi-\pi)$. So, the zero-th Fourier coefficients of $A$ and $B$ are equal respectively to $a_{0} \sigma$ and $b_{0} \sigma$ for all $\xi$ outside the poles of $\sigma(2 i \xi-\pi)$, and all the Fourier coefficients with the negative indices are zero on the whole complex plane of $\xi$. This implies the statement of the Lemma concerning the representation (75). The applicability of (76) can be extended in the same way. q.e.d.

\subsection{Estimates of the solution $m$}

Here, we just list some estimates for $m(z)$ and $m^{\prime}(z)$ immediately following from the asymptotic representations (75) and (76). These estimates will be used in the next section. One has

$$
|m(z)| \leq C P(z), \quad\left|m^{\prime}(z)\right| \leq C(1+|y|) P(z)
$$

where

$$
P(z)=e^{-|y| / 2}\left\{\begin{array}{cc}
e^{|x-\pi-\varphi| y / h}, & y \geq 0 \\
e^{|x+\varphi||y| / h} e^{-\pi|y| / h}, & y \leq 0
\end{array}\right.
$$

where $x=\operatorname{Re} z, y=\operatorname{Im} z$ and $\varphi=\operatorname{Im} \xi$. These estimates are uniform in $\operatorname{Re} z$ if $|\operatorname{Re} z|$ is bounded by a fixed constant.

\section{Minimal solutions of a perturbed auxiliary equa- tion}

In this section, we consider the equation

$$
\psi(z+h)+\psi(z-h)+2 e^{\xi} \cos z \psi(z)=w(z) \psi(z),
$$

assuming that $\xi$ is complex number, and $w$ is a meromorphic function satisfying the estimate

$$
|w(z)| \leq C e^{(1-\mu)|y|}, \quad 0<\mu, \quad|y|>Y,
$$

for sufficiently big $Y$. Here, as below, in this section, we use the notations

$$
x=\operatorname{Re} z, \quad y=\operatorname{Im} z .
$$

Our aim is to prove the existence of a meromorphic solution of (90) having the asymptotics representations of the same form as ones of the minimal solution $m$ of equation (56).

Remind that $m$ has poles if $\sigma(2 i \xi-\pi)=0$, e.i. if

$$
2 i \xi=2 \pi+h+2 h l+2 \pi m, \quad l, m=0,1,2, \ldots
$$


Let

$$
\Sigma=\{ \pm(2 \pi+h+2 h l+2 \pi m), l, m=0,1,2, \ldots\} .
$$

In this section, we assume that

$$
2 i \xi \notin \Sigma \text {. }
$$

One can try to invert the operator being in the left-hand side (89). As we shall see, this leads to an integral equation of the form

$$
\psi(z)=m(z)+\int_{\gamma} \kappa(z, \zeta) \psi(\zeta) d \zeta, \quad z \in \gamma
$$

Here $\gamma$ is a vertical curve, and $\kappa$ is constructed in terms of $m$ and $\tilde{m}$ two linearly independent solutions of (56),

$$
\kappa(z, \zeta)=\frac{1}{2 i h} \theta(z, \zeta) \frac{[m(z) \tilde{m}(\zeta)-m(\zeta) \tilde{m}(z)]}{\{m, \tilde{m}\}} w(\zeta)
$$

where

$$
\theta(z, \zeta)=\operatorname{ctg} \frac{\pi(\zeta-z)}{h}+i
$$

and $\{m, \tilde{m}\}=m(z+h) \tilde{m}(z)-m(z) \tilde{m}(z+h)$ is the wronskian of $m$ and $\tilde{m}$. This integral equation is our main tool. Note that the kernel $\kappa$ can be considered as a difference analog of the resolvent kernel arising in the theory of differential equations: now, instead of the canonical $\theta$-function, $\theta(y-\eta)$, which equals to zero if $y>\eta$ and to 1 if $y<\eta$, one encounters the function (93).

First, we shall study the integral equation (92), and then we shall study its solutions and, in particular, check that they satisfy (89). The analysis of the integral equation is quite similar to one we have carried out in the case of Harper equation $\psi(z+h)+\psi(z-h)+2 \cos z \psi(z)=E \psi(z), E=$ Const, in [8]. So, we describe in details only its new elements.

The main results concerning (89) are formulated in subsection 4.4.

\subsection{Operator $K$}

Now, we are going to describe precisely the construction of the integral operator from (92) (the solutions $m$ and $\tilde{m}$, the integration contour), to describe the functional space and to discuss properties of this operator.

\subsubsection{The solutions $m$ and $\tilde{m}$}

Let $m$ be the minimal solution of (56) constructed in Section 3 , put

$$
\tilde{m}(z, \xi)=\overline{m(\bar{z}, \bar{\xi})} \text {. }
$$


Here, we indicated explicitly the dependence of $m$ on $\xi$. Together with $m, \tilde{m}$ is an entire in $z$ solution of (56).

Remind that $m$ is analytic in $\xi$ outside the set of zeros of $\sigma(2 i \xi-\pi)$. Thus, both $m$ and $\tilde{m}$ are analytic under the condition (91).

The new solution has the asymptotics

$$
\begin{gathered}
\tilde{m}(z)=\left(\bar{c}_{0}(\bar{\xi})+o(1)\right) e^{-\frac{i}{2 h}(z-\pi+i \xi)^{2}+\frac{i z}{2}}+ \\
+e^{\frac{2 \pi i}{h} z}\left(\bar{d}_{0}(\bar{\xi})+o(1)\right) e^{\frac{i}{2 h}(z-\pi+i \xi)^{2}+\frac{i z}{2}}, \\
z \rightarrow+i \infty,
\end{gathered}
$$

and

$$
\begin{gathered}
\tilde{m}(z)=\left(\bar{a}_{0}(\bar{\xi})+o(1)\right) e^{-\frac{i}{2 h}(z-\pi-i \xi)^{2}-\frac{i z}{2}}+ \\
\left(\bar{b}_{0}(\bar{\xi})+o(1)\right) e^{\frac{i}{2 h}(z-\pi-i \xi)^{2}-\frac{i z}{2}} \\
z \rightarrow-i \infty .
\end{gathered}
$$

Here, $a_{0}, b_{0}, c_{0}$ and $d_{0}$ are the coefficients from the asymptotic formulae for $m$.

\subsubsection{The wronskian of $m$ and $\tilde{m}$}

When proving Lemma 3.3, we have already discussed the space of solutions of equation (56), the linear independence and the wronskians of its solutions. We calculate the wronskian of $m$ and $\tilde{m}$ as we have calculated wronskians in the proof of Lemma 3.3. We recall that the wronskian is $h$-periodic and get its asymptotics for $z \rightarrow \pm i \infty$ (using the asymptotics of $m$ and $\tilde{m}$ ). This leads to the result

$$
\{m(z), \tilde{m}(z)\}=e^{\xi} \bar{c}_{0}(\bar{\xi}) a_{0}(\xi) .
$$

This and formulae (77) - (79) imply

$$
\{m(z), \tilde{m}(z)\}=-4 \pi i h e^{\xi / 2} .
$$

Note that this result means that the solutions $m$ and $\tilde{m}$ are linearly independent over the ring of $h$-periodic in $z$ functions.

\subsubsection{The integration contour}

Let $\varphi=\operatorname{Im} \xi$. The contour $\gamma$ is a strictly vertical curve which does not pass through any pole of $w$, comes from $-i \infty$ along the line

$$
x=-\varphi, \quad,
$$


and goes to $+i \infty$ along the line

$$
x=\pi+\varphi .
$$

To motivate this specific choice of the asymptotes of $\gamma$, note that the estimates (88) show that, $|m|$ is minimal along $\gamma$ :

$$
\begin{gathered}
|m(z)| \leq C e^{-|y| / 2} \frac{1}{p_{0}(z)}, \quad z \in \gamma, \\
p_{0}(z)=\left\{\begin{array}{cc}
1, & y>0 \\
e^{\pi|y| / h}, & y \leq 0
\end{array}\right.
\end{gathered}
$$

Note also that

$$
|\tilde{m}(z)| \leq C e^{-|y| / 2} p_{0}(z), \quad z \in \gamma
$$

\subsubsection{The functional space}

We define the operator $K$ as the integral operator with the kernel (93) acting in the space $L_{2}(\gamma, p)$, where $p$ is the weight reflecting as the behavior of $m$ and $\tilde{m}$ along $\gamma$, so the one of $w$, see (90),

$$
p(z)=e^{(1-\mu)|y|} p_{0}^{2}(z) .
$$

\subsubsection{Compactness of the operator $K$}

The kernel of the operator $K$ is quickly decaying along the contour $\gamma$ : using the estimates (87) for $m$ and $m^{\prime}$, one can show that

$$
p^{1 / 2}(z)|\kappa(z, \zeta)| p^{-1 / 2}(\zeta) \leq C(1+|\eta|) e^{-\frac{\mu}{2}|y|-\frac{\mu}{2}|\eta|}, \quad \eta=\operatorname{Im} \zeta, \quad z, \zeta \in \gamma
$$

The estimate (105) implies that the operator $K$ is a Hilbert-Schmidt operator in $L_{2}(\gamma, p)$.

\subsection{Solutions of equation (89) in a vicinity of the curve $\gamma$}

Note that, $m \in L_{2}(\gamma, p)$. Therefore, at least, one of the equations

$$
\psi=m+K \psi \quad \text { and } \quad \psi=K \psi,
$$

has a nontrivial solution $\psi \in L_{2}(\gamma, p)$. In the sequel, $\psi$ denotes this nontrivial solution. If both the equations have nontrivial solutions from $L_{2}(\gamma, p)$, then $\psi$ is one of them. Show that $\psi$ can be analytically continued into a vicinity of $\gamma$ up to a solution of equation (89). 


\subsubsection{Analytic continuation}

One has

Proposition 4.1. There exists a positive $\delta$, such that $\psi$ can be analytically continued in the horizontal $(h+\delta)$-vicinity of $\gamma$.

Proof. For $\zeta \in \gamma$, the kernel $\kappa(z, \zeta)$ is analytic in $z$ in the horizontal $h$-vicinity $S_{h}$ of $\gamma$. The integral, representing $K \psi$, converges uniformly in $z$ being in any compact subset of $S_{h}$. Therefore, the function $K \psi$ can be analytically continued in $S_{h}$. But then, $\psi$, being a solution of (106), can be also analytically continued in $S_{h}$.

Let $\delta$ be the horizontal distance from $\gamma$ to the closest to it pole of the function $w$. If $\delta<h$, then having proved that $\psi$ is analytic in the horizontal $h$-vicinity of $\gamma$, one can deform the integration contour in the formula for $K \psi$ inside its horizontal $\delta$-vicinity, and check that, in fact, $\psi$ can be analytically continued in the horizontal $h+\delta$-vicinity. If $\delta>h$, then one deforms the contour inside its horizontal $h$-vicinity to check that $\psi$ is analytic in $2 h$-vicinity of $\gamma$ and so on.

q.e.d.

For brevity, we shall not distinguish $\psi$ and its analytic continuation.

\subsection{2 $\psi$ and equation (89)}

Note that the horizontal width of the horizontal $(h+\delta)$-vicinity $S_{h+\delta}$ of $\gamma$ is bigger than $2 h$. Show that $\psi$ satisfies equation (89) for $z-h, z, z+h \in S_{h+\delta}$. but

Let $\left(\mathcal{H}_{0} f\right)(z)=f(z+h)+f(z-h)+2 e^{\xi} \cos z f(z)$. Then, $\mathcal{H}_{0} \psi=\mathcal{H}_{0} K \psi$,

$$
\begin{gathered}
\left(\mathcal{H}_{0} K \psi\right)(z)=2 \pi i \operatorname{Res}_{\zeta=z} \kappa(z+h, \zeta) \psi(\zeta)= \\
=\frac{(m(z+h) \tilde{m}(z)-m(z) \tilde{m}(z+h))}{\{m, \tilde{m}\}} w(z) \psi(z)=w(z) \psi(z) .
\end{gathered}
$$

Thus, we come to

Proposition 4.2. The solution $\psi$ satisfies equation (89) in the strip $S_{h+\delta}$.

\subsubsection{Asymptotics of $\psi$ for $z \rightarrow+i \infty$}

Let us describe the asymptotic behavior of $\psi$ in a vicinity of $\gamma$. We begin by proving 
Proposition 4.3. Fix positive $\varepsilon_{1}$ and $\varepsilon_{2}$ so that $\varepsilon_{1}<2 h$ and $\varepsilon_{2}<\min \{\mu, 2 \pi / h\}$. In the horizontal $\varepsilon_{1}$-vicinity of $\gamma$, the solution $\psi$ admits the asymptotic representation

$$
\psi(z)=m(z)\left(A+A_{1}(z)\right)+\tilde{m}(z)\left(B+B_{1}(z)\right), \quad z \rightarrow+i \infty
$$

where

$$
A=\delta(\psi)+\frac{1}{W h} \int_{\gamma} \tilde{m}(\zeta) w(\zeta) \psi(\zeta) d \zeta, \quad B=-\frac{1}{W h} \int_{\gamma} m(\zeta) w(\zeta) \psi(\zeta) d \zeta
$$

and the functions $A_{1}$ and $B_{1}$ satisfy the estimates

$$
\left|A_{1}(z)\right|,\left|B_{1}(z)\right| \leq C e^{-\varepsilon_{2} y}, \quad y>Y,
$$

for sufficiently big $Y$, finally, $W=\{m, \tilde{m}\}$, and

$$
\delta(\psi)=\left\{\begin{array}{l}
1, \quad \text { if } \quad \psi=m+K \psi \\
0, \quad \text { if } \psi=K \psi
\end{array}\right.
$$

Remark. Note that the form of the above asymptotic representation depends on the integral equation for $\psi$.

The proof of this statement is almost the same as the proof of Proposition 3.6 from [8]. Here, we just outline the main idea of the proof. The function $\psi$ satisfies one of the equations from (106). Thus, on the contour $\gamma$, one can estimate it by estimating the right hand side of the equation. Using the inclusion $\psi \in L_{2}(\gamma, p)$ and the estimate (105), one obtains

$$
|\psi(z)| \leq \text { Const } e^{-|y / 2|} / p_{0}(z) .
$$

The integrals

$$
\int_{\gamma} m(\zeta) w(\zeta) \psi(\zeta) d \zeta, \quad \int_{\gamma} \tilde{m}(\zeta) w(\zeta) \psi(\zeta) d \zeta
$$

quickly converge as $\zeta \rightarrow+i \infty$, and one can obtain the asymptotics of $K \psi(z)$ for $z \rightarrow+i \infty$ inside the horizontal $h$-vicinity of $\gamma$ by replacing in the kernel of $K$ the function $\theta(z, \zeta)$ with $2 i$. This leads to the answer

$$
\psi(z) \sim A m(z)+B \tilde{m}(z) .
$$

We omit the elementary estimates leading to (109). To prove (107) outside the horizontal $h$-vicinity of $\gamma$, one has to write down a convenient representation for the analytic continuation of $K \psi$ outside $\gamma$. For example, if $z$ is to the right of $\gamma$, and the horizontal distance between $z$ and $\gamma$ is between $h$ and $2 h$, one can use the formula

$$
(K \psi)(z)=2 \pi i \operatorname{Res}_{\zeta=z-h} \kappa(z, \zeta) \psi(\zeta)+\int_{\gamma} \kappa(z, \zeta) \psi(\zeta) d \zeta
$$


q.e.d.

Using the representation (107) for $\psi$ and the asymptotics (75) $-(76)$ for $m$, one finally obtains the asymptotics of $\psi$ for $z$ being in the horizontal $\varepsilon_{1}$-vicinity of $\gamma$ :

$$
\begin{aligned}
\psi(z)=(a+ & o(1)) e^{\frac{i}{2 h}(z-\pi+i \xi)^{2}+\frac{i z}{2}}+ \\
& +(b+o(1)) e^{-\frac{i}{2 h}(z-\pi+i \xi)^{2}+\frac{i}{2} z}, \quad z \rightarrow+i \infty
\end{aligned}
$$

with

$$
a=A a_{0}(\xi), \quad b=A b_{0}(\xi)+B \overline{c_{0}(\bar{\xi})}
$$

where $a_{0}, b_{0}$ and $c_{0}$ are the coefficients from the asymptotic representations (75)(76) for $m(z)$.

\subsubsection{Asymptotics of $\psi$ for $z \rightarrow-i \infty$}

Describing the behavior of $\psi$ for $z \rightarrow-i \infty$, one proves first the representation $\psi(z)=\left(C+C_{1}(z)\right) m(z)+\left(D+D_{1}(z)\right) e^{-\frac{2 \pi i}{h} z} \tilde{m}(z), \quad z \in S_{\varepsilon_{1}}(\gamma), \quad \operatorname{Im} z \rightarrow-\infty$

Here, $C_{1}$ and $D_{1}$ are functions satisfying the estimates of the form (109), and $C$ and $D$ are constant coefficients given by

$$
C=\delta(\psi), \quad D=-\frac{2}{W h} \int_{\gamma} e^{+2 \pi i \zeta} m(\zeta) w(\zeta) \psi(\zeta) d \zeta
$$

Remark. Let us emphasize that, now, the asymptotic representation strongly depends on the integral equation for $\psi$ : if $\psi$ is a solution of the inhomogeneous equation, then $C=1$; if $\psi$ is a solution of the homogeneous equation, then $C=0$.

The representation (113) implies that, in the horizontal $\varepsilon_{1}$-vicinity of $\gamma$,

$$
\begin{aligned}
\psi(z)=(c & +o(1)) e^{\frac{i}{2 h}(z-\pi-i \xi)^{2}-\frac{i z}{2}}+ \\
& e^{-\frac{2 \pi i}{h} z}(d+o(1)) e^{-\frac{i}{2 h}(z-\pi-i \xi)^{2}-\frac{i}{2} z}, \quad z \rightarrow-i \infty
\end{aligned}
$$

with

$$
c=C c_{0}(\xi), \quad d=C d_{0}(\xi)+D \overline{a_{0}(\bar{\xi})} .
$$

where $a_{0}, c_{0}$ and $d_{0}$ are the coefficients from the asymptotic representations (75) (76) for $m(z)$. 


\subsection{Solution $\psi$ outside the vicinity of $\gamma$}

\subsubsection{Analyticity near $\pm i \infty$}

Being analytic in the horizontal $h+\delta$-vicinity of $\gamma$, the function $\psi$ can be meromorphically continued into the whole complex plane just by means of equation (89). Since $w$ is analytic in some vicinities $\mathbb{C}_{ \pm}$of $\pm i \infty, \psi$ is also analytic there.

\subsubsection{Asymptotics in $\mathbb{C}_{ \pm}$}

Discuss the asymptotics of $\psi$ as $y \rightarrow \pm \infty$ for arbitrary fixed $x$. Begin with the case of $z \rightarrow+i \infty$. As for equation (56), see subsection 3.4.3, one can construct for (89) the canonical basis solutions $f_{1,2}$ analytic in a vicinity $\mathbb{C}_{+}$of $+i \infty$. This can be carried in the same way as in the proof of Theorem 1.1. One obtains

$$
f_{1,2}(z)=e^{ \pm \frac{i}{2 h}(z-\pi+i \xi)^{2}+\frac{i}{2} z}(1+o(1)), \quad y \rightarrow+i \infty
$$

In $\mathbb{C}_{+}$, one can represent $\psi$ by their linear combination with $h$-periodic analytic coefficients

$$
\psi(z)=A(z) f_{1}(z)+B(z) f_{2}(z)
$$

Now, we recall that the asymptotic representation (111) is valid in the $\varepsilon_{1}$-vicinity of $\gamma$ and that as $\varepsilon_{1}$ one can be choose any number from $(0,2 h)$. In particular, this representation is valid in a horizontal vicinity of the horizontal length bigger then $h$, i.e. the period of $A$ and $B$. Therefore, comparing this representation with (111), we see that the periodic coefficients $A$ and $B$ are bounded as $z \rightarrow+i \infty$, and

$$
A(z) \rightarrow a, \quad B(z) \rightarrow b, \quad z \rightarrow+i \infty .
$$

Now, as $A$ and $B$ are $h$-periodic, (115) - (117) imply that the asymptotic representation (111) remains valid and uniform in $\operatorname{Re} z$ if $\operatorname{Im} z \rightarrow+i \infty$ and $|\operatorname{Re} z|$ is bounded by any fixed constant.

Reasoning in the same way one proves that the asymptotic representation (114) for $\psi$ as $y \rightarrow-i \infty$ remains valid and (locally) uniform in $\operatorname{Re} z$ too.

\subsection{Results of the section}

The main results of this section can be formulated in the following form.

Proposition 4.4. Let $2 i \xi \notin \Sigma$. Let also $w$ be a meromorphic function satisfying the estimate (90). Assume that $\gamma$ is a strictly vertical curve not passing through any pole of $w$. Then equation (89) has a meromorphic solution $\psi(z)$ with the following properties: 
1. $\psi$ is analytic in $z$ in some vicinities $\mathbb{C}_{ \pm}$of $\pm i \infty$;

2. there is a positive $\delta$ such that $\psi$ has no poles in the horizontal $(h+\delta)$-vicinity of $\gamma$;

3. $\psi$ admits the asymptotic representations of the form

$$
\begin{aligned}
\psi(z, \xi)=(a+ & o(1)) e^{\frac{i}{2 h}(z-\pi+i \xi)^{2}+\frac{i z}{2}}+ \\
& +(b+o(1)) e^{-\frac{i}{2 h}(z-\pi+i \xi)^{2}+\frac{i}{2} z}, \quad y \rightarrow+\infty
\end{aligned}
$$

and

$$
\begin{aligned}
\psi(z, \xi)= & (c+o(1)) e^{\frac{i}{2 h}(z-\pi-i \xi)^{2}-\frac{i z}{2}}+ \\
& e^{-\frac{2 \pi i}{h} z}(d+o(1)) e^{-\frac{i}{2 h}(z-\pi-i \xi)^{2}-\frac{i}{2} z}, \quad y \rightarrow-\infty
\end{aligned}
$$

where $a, b, c$, and $d$ are independent of $z$. The error estimates are uniform in $x$ if $|x|$ is bounded by a constant.

Remark. The solution $\psi$ satisfies one of the integral equations (106), if it satisfies the homogenous equation, then the coefficient $c$ in the asymptotic representation (119) is zero.

\section{Minimal entire solutions of equation (1)}

In this section, we construct the minimal entire solutions of the matrix equation (1) and prove Theorem 1.2.

\subsection{The plan}

We start with the equation (5) for the first component of a vector solution of (1). We represent $\psi_{1}$ in the form

$$
\psi_{1}(z)=t(z) f_{0}(z)
$$

where $t$ satisfies the equation

$$
t(z+h)=\rho(z) t(z-h)
$$

This transforms (5) to

$$
f_{0}(z+h)+f_{0}(z-h)=v_{1}(z) f_{0}(z)
$$

with

$$
v_{1}(z)=\frac{t(z)}{t(z+h)} v(z) .
$$


We find a solution $t$ of (121) such that $t(z) / t(z+h)$ tends to some nonzero constants as $z \rightarrow \pm i \infty$.

Then, we accept assumption (21). In this case,

$$
v_{1}(z) \sim v_{1-} e^{i n z}+v_{1+} e^{-i n z}, \quad z \rightarrow \pm i \infty
$$

and, by a linear change of the variable, one transforms (122) to equation (89) investigated in the previous section. This allows to construct some analytic solution $\psi_{1}$ of equation (5). This function can be considered as the first component of a vector solution $\psi$ of the original matrix equation (1). The second component can be reconstructed by formula (6).

To be sure that $\psi$ is really entire in $z$, we check that it is analytic in a sufficiently wide horizontal vicinity a vertical curve $\gamma$. Really, since $\operatorname{det} M(z) \equiv 1$, the formulae

$$
\psi(z+h)=M(z) \psi(z), \quad \psi(z-h)=M^{-1}(z-h) \psi(z)
$$

show that, in this case, $\psi$ can be continued up to an entire function of $z$.

\subsection{Meromorphic solution of equation (121)}

Let $\gamma$ be a strictly vertical curve. Here, we construct a meromorphic solution of (121) analytic in a vicinity of $\gamma$. We shall use the following notations:

- we denote by $\gamma+2 \pi$ the curve obtained of $\gamma$ by the $2 \pi$-translation;

- we denote by $S_{\gamma}$ the strip bounded by $\gamma$ and $\gamma+2 \pi$ so that $\gamma \subset S_{\gamma}$ and $\{\gamma+2 \pi\} \cap S_{\gamma}=\emptyset$

- we let $n_{ \pm}=n_{ \pm}(b)$ and $N=n_{+}+n_{-}$;

- we denote the zeros of $b(z)$ situated in $S_{\gamma}$ by $z_{l}, l=1,2, \ldots N$.

Proposition 5.1. Let $\gamma$ be a strictly vertical curve not passing through any point where either $b(z)=0$ or $b(z-h)=0$. There exists a meromorphic solution $t$ of equation (121) and a positive number $\delta$ such that

(i) $t$ is analytic in the horizontal $(h+\delta)$-vicinity of $\gamma$;

(ii) if $z_{0}$ is a zero of $b(z)$ situated in this vicinity to the left of $\gamma$, then $t\left(z_{0}\right)=0$; in addition, the multiplicity of the zero of $t$ equals to the multiplicity of the zero of $b(z)$ at $z=z_{0}$; $\gamma$

(iii) the function $v_{1}$ defined by (123) is analytic in the horizontal $\delta$-vicinity of 
(iv) the function $t$ admits the asymptotic representations

$$
\begin{gathered}
t(z)=e^{i n_{-} z / 2}\left(1+\tau_{-}(z)\right), \quad \operatorname{Im} z \rightarrow-\infty \\
t(z)=t_{\infty} e^{-i n_{+} z / 2}\left(1+\tau_{+}(z)\right), \quad \operatorname{Im} z \rightarrow+\infty
\end{gathered}
$$

where

$$
t_{\infty}=\exp \left(\frac{i}{2} \sum_{l=1}^{n_{+}+n_{-}} z_{l}-i \pi N / 2+i h N / 4\right),
$$

and $\tau_{ \pm}$are functions satisfying the estimates

$$
\left|\tau_{ \pm}(z)\right| \leq C e^{-\mu|\operatorname{Im} z|},
$$

where $\mu$ is a positive number. This formulae are uniform in $\operatorname{Re} z$ if $|\operatorname{Re} z|$ is bounded by a fixed constant.

Proof. 1. Construct a solution of (121) in terms of the $\sigma$-function introduced in subsection 3.2. The function $\rho(z)=b(z) / b(z-h)$ can be represented in the form

$$
\rho(z)=e^{i n_{-} h} \prod_{l=1}^{N} \rho_{l}(z), \quad \rho_{l}(z)=\frac{1-e^{-i\left(z-z_{l}\right)}}{1-e^{-i\left(z-z_{l}-h\right)}} .
$$

Substituting this representation in equation (121), and comparing the result with (59), one immediately finds out that the function

$$
t(z)=e^{i n_{-} z / 2} \prod_{l=1}^{N} t_{l}(z), \quad t_{l}(z)=\frac{\sigma\left(z+\pi-z_{l}\right)}{\sigma\left(z+\pi-z_{l}-h\right)},
$$

is a solution of (121). Clearly, it is meromorphic in $z$.

2. To prove the proposition, we have to recall some properties of the $\sigma$-function. First, we note that the zeros of the $\sigma$-function are situated at the points $\pi+h+$ $2 h l+2 \pi m, l, m \in \mathbb{N} \cup\{0\}$, and that its poles are at the points $-\pi-h-2 h l-2 \pi m$, $l, m \in \mathbb{N} \cup\{0\}$. Secondly, we remind that as the pole at $z=-\pi-h$ so the zero at $z=\pi+h$ are simple. Moreover, if $2 \pi m<2 h, m \in \mathbb{N}$, then all the poles and zeros situated at the points $z=-\pi-h-2 \pi l$, and $z=\pi+h+2 \pi l, l=0, \ldots m$, are simple.

The ratio $\frac{\sigma(z+\pi)}{\sigma(z+\pi-h)}$ has poles only at the points

$$
z=2 h+2 h l+2 \pi m, \quad \text { and } \quad-2 \pi-h-2 h l-2 \pi m, \quad l, m \in \mathbb{N} \cup\{0\} .
$$

The zeros of this ratio are at the points

$$
z=h+2 h l+2 \pi m, \quad \text { and } \quad-2 \pi-2 h l-2 \pi m, \quad l, m \in \mathbb{N} \cup\{0\},
$$


the zeros at $z=h$ and at $z=-2 \pi$ are simple. Moreover, if $2 \pi m<2 h, m \in \mathbb{N}$, then all the zeros situated at the points $z=h+2 \pi l$ and $z=-2 \pi-2 \pi l, l=0, \ldots m$, are simple.

Now, we shall show that the described properties of the ratio $\frac{\sigma(z+\pi)}{\sigma(z+\pi-h)}$ imply the first three statements of the proposition.

3. Show that there is a positive number $\delta$ such that the constructed $t$ is analytic in the horizontal $h+\delta$-vicinity of $\gamma$.

Denote by $\delta_{1}$ the horizontal distance between the set of the points $z_{l}, l=1, \ldots, N$, and the curve $2 \pi+\gamma$. The poles of the factor $t_{l}(z)$ closest to $\gamma$ are at the points $z_{l}+2 h$ and $z_{l}-2 \pi-h$. So, it is analytic in the horizontal $h+\delta$-vicinity of $\gamma$, where

$$
\delta=\min \left\{\delta_{1}, h\right\}
$$

This implies the first statement of the proposition.

4. Let us prove the second statement. Let $z_{0}$ be a zero of $b(z)$ situated to the left of the curve $\gamma$ inside the above horizontal $h+\delta$-vicinity of $\gamma$. Show that $t\left(z_{0}\right)=0$.

All the factors $t_{l}$ are analytic in the vicinity of $\gamma$. Inside this vicinity, to the left of $\gamma$, the factor $t_{l}$ equals to zero only at the points $z_{l}-2 \pi m, m=1,2, \ldots$ This proves the first part of the second statement. Prove the second part.

The choice of $\delta$ implies that a point of the form $z_{l}-2 \pi m, m=2,3,4, \ldots$, can be situated in the horizontal $(h+\delta)$-vicinity of $\gamma$ only if $2 \pi m<h$. So, all the zeros of $t_{l}$ being in this vicinity to the left of $\gamma$ are simple. In result, the multiplicity of the zero of $t(z)$ at any of these points being in the vicinity equals to the number of the factors $t_{l}$ which are equal to 0 there, e.i. to the multiplicity of the zero of $b$ at this point.

5. The function $v_{1}(z)$ defined by (123) is clearly meromorphic. It can be also represented in the form

$$
v_{1}(z)=\frac{t(z)}{t(z+h)} a(z)+\frac{t(z)}{t(z-h)} d(z-h) .
$$

Formula (131) shows that the poles of $v_{1}$ situated in the above horizontal vicinity of $\gamma$ can be only at the points where $t(z+h)=0$ or (and) $t(z-h)=0$, e.i. at the points

$$
z=z_{l}+2 h l+2 \pi m, \quad \text { and } \quad z=z_{l}-2 \pi+h-2 h l-2 \pi m, \quad l, m \in \mathbb{N} \cup\{0\} .
$$

Among them, only the points

$$
z_{l}+2 \pi m \text { and } z_{l}-2 \pi m+h, \quad m=0,1, \ldots,
$$


can be in the above vicinity. So, they can be only the points where either $b(z)=0$ or $b(z-h)=0$. However, the curve $\gamma$ does not pass through any of these points. Thus, there is a positive $\delta_{2}$ such that the function $v_{1}$ is analytic in the horizontal $\delta_{2}$-vicinity of $\gamma$. If this $\delta_{2}$ is smaller than the previously defined $\delta$, we simply redefine $\delta=\delta_{2}$.

6. The asymptotics of $t$ for $z \rightarrow \pm i \infty$ follow immediately from formula (130) and the asymptotics (64) and (65) of the $\sigma$-function.

q.e.d.

\subsection{Entire solutions of (1)}

Here, following the plan described above, we shall construct a set of entire solutions of (1).

1. Let $\gamma$ be a strictly vertical curve do not passing through any point where either $b(z)=0$ or $b(z-h)=0$, and let $t$ be the solution of equation (121) described in Proposition 5.1. Let us study in more details the function $v_{1}$ defined by (123).

By Proposition 5.1, $v_{1}$ is analytic in the $\delta$-vicinity of $\gamma$. In the case of $(21)$, the asymptotic representations for $t$ imply that

$$
v_{1}(z)=-e^{i\left(n z+\phi_{-}+\pi\right)}-e^{-i\left(n z+\phi_{+}+\pi\right)}+w(z),
$$

where

$$
\phi_{-}=-n_{-}(b) h / 2-i \ln v_{-}, \quad \phi_{+}=-n_{+}(b) h / 2+i \ln v_{+},
$$

and $w$ is a function satisfying the estimate

$$
|w(z)| \leq C e^{(n-\mu)|\operatorname{Im} z|}, \quad|\operatorname{Im} z|>Y,
$$

where $\mu$ is the same number as in (128), and $Y$ is a sufficiently big positive number. This estimate is uniform in $\operatorname{Re} z$ if $|\operatorname{Re} z|$ is bounded by a constant.

2. Let

$$
\phi=\left(\phi_{+}+\phi_{-}\right) / 2, \quad 2 i \xi=\phi_{+}-\phi_{-} .
$$

Equation (122) can be rewritten in the form:

$$
f_{0}(z+h)+f_{0}(z-h)+2 e^{\xi} \cos (n z+\phi+\pi) f_{0}(z)=w(z) f_{0}(z) .
$$

The change of the variable

$$
z_{1}=n z+\phi+\pi
$$


transforms (135) to the equation

$$
\psi\left(z_{1}+h_{1}\right)+\psi\left(z_{1}-h_{1}\right)+2 e^{\xi} \cos \left(z_{1}\right) \psi\left(z_{1}\right)=w_{1}\left(z_{1}\right) \psi\left(z_{1}\right)
$$

where

$$
\psi\left(z_{1}\right)=f_{0}(z), \quad w_{1}\left(z_{1}\right)=w(z), \quad h_{1}=n h .
$$

This equation was investigated in the previous section.

3. Now, we can construct a solution of (135) by means of Proposition 4.4.

Check that the coefficients of (137) satisfy its assumptions. The estimate of $w(z)$ implies that $w_{1}$ satisfies an estimate of the form (90) as a function of $z_{1}$. Note that the branches of the logarithms in (133) are fixed modulo $2 \pi$. To ensure the assumption on $\xi$, we fix them so that the parameters $\phi_{ \pm}$satisfy condition (18).

To apply the proposition, it rests to choose a vertical curve mentioned in it. We denote this curve $\gamma_{1}$ and choose it so that it be the image under the transformation $z \mapsto z_{1}$ of the curve $\gamma$ used for constructing of the function $t$. To apply the proposition, we have to check that $\gamma_{1}$ does not not pass by any pole of $w$. But this follows from the statement (iii) of Proposition 5.1.

Now, we construct the solution $\psi$ of (137) by means of Proposition 4.4. Note that this solution is analytic in the horizontal $\left(h_{1}+\delta_{1}\right)$-vicinity of $\gamma_{1}$, where $\delta_{1}$ is a positive number. This number is determined by the horizontal distance between the poles of $w_{1}$ and the curve $\gamma_{1}$. We can assume that $\delta_{1}=n \delta$ ( if $\delta_{1}>n \delta$, we redefine it letting $\delta_{1}=n \delta$; if $\delta_{1}<n \delta$, we redefine $\delta$ ).

Indicating explicitly the dependence of $\psi$ on $\xi$ and $h$, we let

$$
f_{0}(z)=\psi\left(n_{0} z+\phi+\pi, \xi, n h\right) \text {. }
$$

The function $f_{0}$ is a solution of (135). It is analytic in the horizontal $h+\delta$-vicinity of $\gamma$.

4. The function $\psi_{1}=t f_{0}$ is a solution of equation (5), and, thus, can be considered as the first component of a vector solution $\Psi$ of the matrix equation (1). Its second component can be reconstructed by formula (6).

5. Let us discuss the analytic properties of $\psi_{1}$.

First, note that the asymptotics of $f_{0}$ (following from Proposition 4.3), and the asymptotics for $t$ described by (125) and (126) imply the representations

$$
\begin{aligned}
\psi_{1}(z) & =\left(A_{0}+o(1)\right) e^{\frac{i}{2 h n}\left(n z+\phi_{+}\right)^{2}+\frac{i z}{2}\left(n-n_{+}\right)_{+}} \\
& +\left(B_{0}+o(1)\right) e^{-\frac{i}{2 h n}\left(n z+\phi_{+}\right)^{2}+\frac{i z}{2}\left(n-n_{+}\right)}, \quad \operatorname{Im} z \rightarrow+\infty
\end{aligned}
$$




$$
\begin{aligned}
& \psi_{1}(z)=\left(C_{0}+o(1)\right) e^{\frac{i}{2 h n}\left(n z+\phi_{-}\right)^{2}-\frac{i z}{2}\left(n-n_{-}\right)}+ \\
& +e^{-\frac{2 \pi i}{h} z}\left(D_{1}+o(1)\right) e^{-\frac{i}{2 h n}\left(n z+\phi_{-}\right)^{2}-\frac{i z}{2}\left(n-n_{-}\right)}, \quad \operatorname{Im} z \rightarrow-\infty
\end{aligned}
$$

where $n_{ \pm}=n_{ \pm}(b)$, and $A_{0}, B_{0}, C_{0}$, and $D_{1}$ are coefficients independent of $z$.

Secondly, note that since both $t$ and $f_{0}$ are analytic in the horizontal $h+\delta$-vicinity of $\gamma$, the function $\psi_{1}$ is also analytic there.

6. Discuss the analyticity of the second component of the vector $\Psi=\left(\begin{array}{l}\psi_{1} \\ \psi_{2}\end{array}\right)$.

For any $\epsilon>0$, we denote by $L_{\gamma}(\epsilon)$ the part of the horizontal $\epsilon$-vicinity of $\gamma$ situated to the left of $\gamma$. Clearly, $\psi_{1}(z+h)-a(z) \psi_{1}(z)$ is analytic in $L_{\gamma}(h+\delta)$. So, if $b$ has no zeros in this domain, $\psi_{2}(z)=\left(\psi_{1}(z+h)-a(z) \psi_{1}(z)\right) / b(z)$ is also analytic there.

7. As the horizontal length of $L_{\gamma}(h+\delta)$ is greater then $h$ and $\Psi$ is analytic here, then we can continue $\Psi$ in the whole complex plane by means of equation (1): we continue $\Psi$ to the right just by means this equation itself, and we continue it to the left by the formula $\Psi(z-h)=M^{-1}(z-h) \Psi(z)$ (remind that $\operatorname{det} M \equiv 1$ ).

We have proved

Proposition 5.2. Assume that $b \not \equiv 0$ and that $v(z)$ satisfies condition (21). By means of formulae (133), choose some numbers $\phi_{ \pm}$satisfying (18). Let $\gamma$ be a strictly vertical curve not passing through any point where either $b(z)=0$ or $b(z-h)=0$. If the curve can be drown so that there would be no zeros of the function $b$ in the domain $L_{\gamma}(h)$, then equation (1) has an entire solution $\Psi$ with the first component admitting the asymptotic representations (139) - (140).

In fact, the solution $\Psi$ described in Proposition 5.2 is one of the main personages of this paper. In subsection 5.5, we shall see that it is a minimal entire solution of equation (1).

\subsection{Modified approach}

In the previous subsection, we have constructed a (minimal) entire solution of equation(1) under a hypothesis on the geometry of the set of zeros of $b(z)$. We were assuming that there is a strictly vertical curve $\gamma$ such that $b(z)$ and $b(z-h)$ have no zeros on $\gamma$ and $b(z)$ has no zeros in $L_{\gamma}(h)$, i.e. in the left part the horizontal $h$-vicinity of $\gamma$. Now, we shall remove this geometric condition. We begin with the case where $b$ has only one simple zero $z_{0} \in L_{\gamma}(h)$. Let us outline the idea. 


\subsubsection{The idea}

Any solution of (5) can be considered as the first component $\psi_{1}$ of a vector solution $\Psi=\left(\begin{array}{l}\psi_{1} \\ \psi_{2}\end{array}\right)$ of equation (1). Its second component can be recovered by (6). If $\psi_{1}$ is analytic in the horizontal $(h+\delta)$-vicinity of a vertical curve $\gamma$ and such that

$$
\psi_{1}\left(z_{0}+h\right)-a\left(z_{0}\right) \psi_{1}\left(z_{0}\right)=0
$$

then the corresponding vector solution $\Psi$ is analytic at least in $L_{\gamma}(h+\delta)$ and, so, it can be analytically continued up to an entire function just by means of (1).

In the case of Proposition 5.2, we have constructed (minimal) entire solutions of (1) in terms of a solution $f_{0}$ of equation (122) analytic in the horizontal $(h+$ $\delta)$-vicinity of $\gamma$, using the formula $\psi_{1}(z)=t(z) f_{0}(z)$. Now, by Proposition 5.1 (statement (ii)),

$$
t\left(z_{0}\right)=0
$$

So, $\psi_{1}=t f_{0}$ appears to be analytic in the horizontal $(h+\delta)$-vicinity of $\gamma$ even if $f_{0}$ is replaced by a solution of (122) having there one (simple) pole situated at $z_{0}$. For the "old" analytic at $z_{0}$ solution $f_{0}$, the condition (141) is not satisfied. The idea is to satisfy this condition by considering instead of the "old" $f_{0}$ linear combinations of the "old" $f_{0}$ and a "new" solution of (122) having one simple pole at $z_{0}$.

Of course, the meromorphic solutions of (122) we are looking for have to possed asymptotic representations for $z \rightarrow \pm i \infty$ of the same analytic structure as $f_{0}$ so that $\psi_{1}$ would have the asymptotics of the form (139) - (140).

To construct such solutions of (122), we shall consider a solution $m\left(z, z_{0}\right)$ of the model equation (56) possessing the analogous properties. Then, we shall plug it into the integral equation (92) in the place of $m(z)$ (the integral operator remains the same as before) and study its solutions.

\subsubsection{The solution $m\left(z, z_{0}\right)$}

We shall use the variable $z$ of the input matrix equation (1). Let $\phi$ and $\xi$ be as in (134). Consider the equation (compare it with (135))

$$
g(z+h)+g(z-h)+2 e^{\xi} \cos \left(z_{1}\right) g(z)=0, \quad z_{1}=n z+\phi+\pi .
$$

The functions $m\left(z_{1}, \xi, h_{1}\right)$ and $\tilde{m}\left(z_{1}, \xi, h_{1}\right), h_{1}=n h$, are solutions of (143). Here, we have indicated explicitly the dependence of these functions on $h$ and $\xi$.

Let

$$
m\left(z, z_{0}\right)=\theta\left(z, z_{0}\right) \frac{\left[m\left(z_{1}\right) \tilde{m}\left(z_{01}+h_{1}\right)-m\left(z_{01}+h_{1}\right) \tilde{m}\left(z_{1}\right)\right]}{\{m, \tilde{m}\}}
$$


where $\{m, \tilde{m}\}$ is the wronskian of $m(z)$ and $\tilde{m}(z)$, see subsection 4.1 .2 ,

$$
z_{01}=n z_{0}+\phi+\pi
$$

and, as before,

$$
\theta\left(z, z_{0}\right)=\operatorname{ctg} \frac{\pi\left(z_{0}-z\right)}{h}+i
$$

Since the wronskian $\{m, \tilde{m}\}$ is constant, and $\theta\left(z, z_{0}\right)$ is $h$-periodic in $z$, the function $m\left(z, z_{0}\right)$ satisfies equation (143).

Let us discuss analytic properties of $m\left(z, z_{0}\right)$. It is analytic outside the points $z=z_{0}+l h, l \in \mathbb{Z}$. As $\left[m\left(z_{01}\right) \tilde{m}\left(z_{01}+h_{1}\right)-m\left(z_{01}+h_{1}\right) \tilde{m}\left(z_{01}\right)\right]=\{m, \tilde{m}\}$, the solution $m\left(z, z_{0}\right)$ has a simple pole at $z=z_{0}$,

$$
\operatorname{Res}_{z=z_{0}} m\left(z, z_{0}\right)=\frac{h}{\pi}
$$

At the point $z=z_{0}+h$, the numerator in (144) equals to zero, and, thus, in fact, $m\left(z, z_{0}\right)$ is analytic here. So, for some positive $\delta, m\left(z, z_{0}\right)$ is analytic in the horizontal $h+\delta$-vicinity of $\gamma$ if $z \neq z_{0}$.

The asymptotics of $m(z)$ and $\tilde{m}(z)$ for $z \rightarrow \pm i \infty$ imply the asymptotic representations for $m\left(z, z_{0}\right)$ :

$$
\begin{aligned}
m\left(z, z_{0}\right) & =A\left(z_{0}\right) e^{\frac{i}{2 h n}\left(n z+\phi_{+}\right)^{2}+\frac{i n z}{2}}(1+o(1))+ \\
& +B\left(z_{0}\right) e^{-\frac{i}{2 h n}\left(n z+\phi_{+}\right)^{2}+\frac{i n z}{2}}(1+o(1)), \quad \operatorname{Im} z \rightarrow+i \infty \\
m\left(z, z_{0}\right) & =e^{-2 \pi i z / h}\left(C\left(z_{0}\right) e^{\frac{i}{2 h n}\left(n z+\phi_{-}\right)^{2}-\frac{i n z}{2}}(1+o(1))+\right. \\
& \left.+D\left(z_{0}\right) e^{-\frac{i}{2 h n}\left(n z+\phi_{-}\right)^{2}-\frac{i n z}{2}}(1+o(1))\right), \quad \operatorname{Im} z \rightarrow-i \infty
\end{aligned}
$$

where $A, B, C$ and $D$ are independent of $z$.

\subsubsection{The integral operator}

To construct the meromorphic $f_{0}$, we shall again use the integral operator from (92), but now, it will be convenient to write it in terms of the variable of the input equation (1). So, now, $\gamma$ is a strictly vertical curve going to $+i \infty$ along the line $\operatorname{Re} z=-\operatorname{Re} \phi_{+} / n$, and from $-i \infty$ along the line $\operatorname{Re} z=-\left(\pi+\operatorname{Re} \phi_{-}\right) / n$. We define the weight $P(z)=e^{(n-\mu)|y|} p_{0}^{2}\left(z_{1}\right)$, where $y=\operatorname{Im} z, \mu$ is the constant from 
the estimate for $w(z)$ from (132), and $p_{0}$ is defined by (102). Both the functions $m($.$) and m\left(., z_{0}\right)$ belong to $L_{2}(\gamma, P)$.

The kernel of the integral operator takes the form

$$
\kappa(z, \zeta)=\frac{1}{2 i h} \theta(z, \zeta) \frac{\left[m\left(z_{1}\right) \tilde{m}\left(\zeta_{1}\right)-m\left(\zeta_{1}\right) \tilde{m}\left(z_{1}\right)\right]}{\{m, \tilde{m}\}} w(\zeta), \quad \zeta_{1}=n \zeta+\varphi+\pi .
$$

Here, $w$ is the same as in (137). The kernel satisfies the estimate $P^{1 / 2}(z)|\kappa(z, \zeta)| P^{-1 / 2}(\zeta) \leq C(1+|\eta|) e^{-\frac{\mu}{2}|y|-\frac{\mu}{2}|\eta|}, \quad y=\operatorname{Im} z, \quad \eta=\operatorname{Im} \zeta, \quad z, \zeta \in \gamma$

The integral operator $K$ with the kernel (147) is compact in the space $L_{2}(\gamma, P)$.

\subsubsection{Auxiliary construction}

To construct the meromorfic $f_{0}$, we use an operator equation slightly different from (106). Before writing down this equation, we shall prove an auxiliary statement motivating this equation. Note that, since $\operatorname{det} M \equiv 1$, the equality $b\left(z_{0}\right)=0$ implies that $a\left(z_{0}\right) \neq 0$. We put

$$
s\left(z_{0}\right)=\frac{\pi}{h} \frac{t\left(z_{0}+h\right)}{t^{\prime}\left(z_{0}\right) a\left(z_{0}\right)} .
$$

One has

Lemma 5.3. Assume that there exists a function $f \in L_{2}(\gamma, P)$ which can be analytically continued in a vicinity of the point $z_{0}+h$, and satisfies the relation

$$
f(z)=K f(z)+s\left(z_{0}\right) m\left(z, z_{0}\right) f\left(z_{0}+h\right)+\hat{m}(z), \quad z \in \gamma,
$$

where either $\hat{m}(z) \equiv m\left(z_{1}\right)$ or $\hat{m}(z) \equiv 0$. Let $\psi_{1}(z)=t(z) f(z)$. This function has the following properties:

(i) it is a solution of (5) analytic in the horizontal $(h+\delta)$-vicinity of $\gamma$, where $\delta$ is a positive number;

(ii) $\psi_{1}$ satisfies the relation (141);

(iii) for $\operatorname{Im} z \rightarrow \pm i \infty$, it admits the asymptotic representations of the form (139) - (140).

Remark. As we shall see from the proof, if the function $f$ satisfying (150) exists, then it satisfies equation (122). It is the needed meromorphic solution of this 
equation. Furthermore, the function $\psi_{1}=t f$ is the first component of the desired (minimal) entire vector solution of equation (1).

Proof. a. Since $f \in L_{2}(\gamma, P)$, and the kerenel of $K$ satisfies the estimate (148), then the term $K f$ is analytic in the horizontal $h$-vicinity of $\gamma$. Therefore, the right hand side of (150) is analytic in this vicinity except the point $z=z_{0}$. This implies that $f$ itself is also analytic there. At the point $z=z_{0}$, this function has a simple pole,

$$
\operatorname{res}_{z=z_{0}} f(z)=\frac{h}{\pi} s\left(z_{0}\right) f\left(z_{0}+h\right)
$$

Let $\delta_{1}$ be the horizontal distance from $z_{0}$ to $\gamma$. Deforming the integration contour in the definition of $K$, one checks that, in fact, $f$ is analytic in the horizontal $\left(h+\delta_{1}\right)$-vicinity of $\gamma$ without the point $z=z_{0}$.

b. Using a calculation analogous to one from subsection 4.2 .2 , by means of the residue theorem, we check that the relation (150) implies that, in the above vicinity of $\gamma, f$ satisfies the equation (135) which is just a different form of writing of (122).

c. Remind that $t$ is analytic in a horizontal $(h+\delta)$-vicinity of $\gamma$, and $t\left(z_{0}\right)=0$. If $\delta>\delta_{1}$, we redefine it letting $\delta=\delta_{1}$. Let $\psi_{1}=t f$. This function is analytic in the whole horizontal $(h+\delta)$-vicinity of $\gamma$. Since $f$ satisfies $(122), \psi_{1}$ is a solution of equation (5).

d. Check that $\psi_{1}$ satisfies the relation (141). Using (151) and (149), we see that

$$
\psi_{1}\left(z_{0}\right)=t^{\prime}\left(z_{0}\right) \operatorname{res}_{z=z_{0}} f(z)=\frac{t\left(z_{0}+h\right)}{a\left(z_{0}\right)} f\left(z_{0}+h\right)=\frac{1}{a\left(z_{0}\right)} \psi_{1}\left(z_{0}+h\right) .
$$

e. Finally, applying the methods of section 4 to equation (150), one obtains the asymptotics of $f$ for $z \rightarrow \pm i \infty$ which leads to (iii).

q.e.d.

\subsubsection{Operator equation}

Now, we turn to constructing a function $f$ satisfying the hypothesis of Lemma 5.3. Since (150) can not be used directly as an equation for a function from $L_{2}(\gamma, P)$, we change the functional space to $\mathcal{H}=L_{2}(\gamma, P) \oplus \mathbb{C}$. For $F \in \mathcal{H}$, we use the notation

$$
F=\left(\begin{array}{c}
f \\
s
\end{array}\right), \quad f \in L_{2}(\gamma, P), \quad s \in \mathbb{C} .
$$

Then we define the operator $\mathcal{K}$ acting in $\mathcal{H}$ by the formula

$$
\mathcal{K} F=\left(\begin{array}{cc}
K f & m\left(z, z_{0}\right) s \\
K f\left(z_{0}+h\right) & m\left(z_{0}+h, z_{0}\right) s
\end{array}\right) .
$$


For $f \in L_{2}(\gamma, P)$, the function $K f(z)$ is analytic in the horizontal $h$-vicinity of $\gamma$, and thus the number $K f\left(z_{0}+h\right)$ is well defined.

One can easily check that the application $f \longrightarrow K f\left(z_{0}+h\right)$ is a linear bounded operator from $L_{2}(\gamma, P)$ to $\mathbb{C}$, which implies that $\mathcal{K}$ is a compact operator together with $K$.

The new integral equation is

$$
\mathcal{K} F+F_{0}=F, \quad F_{0}(z)=\left(\begin{array}{c}
\hat{m}(z) \\
\hat{m}\left(z_{0}+h\right)
\end{array}\right) .
$$

We let $\hat{m}(z) \equiv m\left(z_{1}\right)$ if $1 \notin \operatorname{spec} \mathcal{K}$ and $\hat{m}(z) \equiv 0$ otherwise.

Clearly, (152) always has a nontrivial solution. For $F$ being a solution of this equation,

$$
\begin{gathered}
f(z)=K f(z)+m\left(z, z_{0}\right) s+\hat{m}(z), \\
s=K f\left(z_{0}+h\right)+m\left(z_{0}+h, z_{0}\right) s+\hat{m}\left(z_{0}+h\right) .
\end{gathered}
$$

Discuss properties of the function $f$.

\subsubsection{The solution of the operator equation and the hypothesis of Lemma 5.3}

Since $f \in L_{2}(\gamma, P)$, it can be analytically continued in a vicinity of $z_{0}+h$ just by means of (153).

Letting in (153) $z=z_{0}+h$ and comparing the result with (154), we see that

$$
s=f\left(z_{0}+h\right) .
$$

Substituting this expression for $s$ in (153), we come to (150).

Thus, we see that $f$ satisfies the assumptions of Lemma 5.3. This means that we have constructed the desired (minimal) entire solution.

\subsubsection{Several zeros of $b$}

We have proved the existence of the minimal solution in the case where $b$ has only one simple zero in the domain $L_{\gamma}(h)$. The case of several zeros (even multiple ones) can be treated similarly. In particular, if, in $L_{\gamma}(h)$, there are $J$ simple zeros of $b$, then instead of (150) one considers the relation

$$
f(z)=K f(z)+\sum_{j=1}^{J} s\left(z_{j}\right) m\left(z, z_{j}\right) f\left(z_{j}+h\right)+\hat{m}(z), \quad z \in \gamma
$$


and instead of $\mathcal{H}$ one has to introduce the space $L_{2}(\gamma, P) \oplus \mathbb{C}^{J}$. We omit the details and formulate the result:

Proposition 5.4. Assume that $b \not \equiv 0$ and that $v(z)$ satisfies condition (21). By means of formulae (133), choose some numbers $\phi_{ \pm}$satisfying (18). Equation (1) has an entire solution $\Psi$ with the first component $\psi_{1}$ admitting the asymptotic representations $(139)-(140)$.

Remark. Note that if $1 \in \operatorname{spec} \mathcal{K}$ then, in the asymptotic representation (140) for $\psi_{1}$, the coefficient $C_{0}$ is zero

\subsection{Minimal entire solutions}

1. Now, let us discuss in more detail the behavior of the solution described in Proposition 5.4 for $z \rightarrow \pm i \infty$. We shall use the canonical basis solutions $f_{1,2}$ and $g_{1,2}$ analytic in some vicinities $\mathbb{C}_{+}$and $\mathbb{C}_{-}$of $+i \infty$ and $-i \infty$ correspondingly, see Theorems $1 \mathrm{a}$ and $1 \mathrm{~b}$.

Remind that any entire solution $\psi$ of (1) admits the representations (19). Consider these representations for the solution $\Psi$ described in Proposition 5.4.

Remind that the asymptotics (10) of the basis Bloch solutions $f_{1,2}$ contain parameter $\phi_{+}$. This parameter is defined by (11) modulo $2 \pi$. The choice of the value of the parameter is a choice of two particular solutions $f_{1,2}$.

Formulae (11) and (133) coincide, and thus, $\phi_{+}$from (10) can be chosen equal to $\phi_{+}$ in (139). In this case, comparing (139) and (10), we see that in a vicinity of $+i \infty$, $\Psi$ admits the first of the representations (19) with $A$ and $B$ bounded as $z \rightarrow+i \infty$.

Choosing the canonical basis $g_{1,2}$ in a vicinity of $-i \infty$ with the same values of $\phi_{-}$ as in (140), one also sees that $\Psi$ admits the second of the representations (19) with $C$ and $D$ bounded as $z \rightarrow-i \infty$, and that, moreover, $D(z) \rightarrow 0$ as $z \rightarrow-i \infty$.

Now, we recall the definition of the minimal entire solutions of (1), and see that the solution $\Psi$ is one of them. It is the solution $\psi_{D}$.

2. The proofs of the existence of the other three minimal solutions are similar to the above one. So, we shall discuss only a place where they essentially differ. This is the integral equation being the starting point for the analysis of Section 4 . Consider for example the case of the solution for which $B(z) \rightarrow 0$ as $z \rightarrow+i \infty$. 
For this solution, equation (92) has to be replaced by

$$
\psi(z)=m(2 \pi-z)+\int_{\{2 \pi-\gamma\}} \kappa(2 \pi-z, 2 \pi-\zeta) \psi(\zeta) d \zeta .
$$

with the same kernel $\kappa$ as in Section 4. The contour $\{2 \pi-\gamma\}$ is obtained of the contour $\gamma$ from Section 4 by the mapping $z \rightarrow 2 \pi-z$. As in Section 4 , constructing a minimal solution of (1), we have used as the first term in the right hand side of the integral equation the minimal solution of equation (56) having the same form of the asymptotics for $z \rightarrow \pm i \infty$ as the solution we construct. The analysis of equation (155) is similar to one of equation (92).

3. We have described how one can construct four minimal entire solutions corresponding to the canonical bases with the given parameters $\phi_{ \pm}$. The only condition on these to numbers is given by (18). In fact, it means that the canonical bases have to be consistent. This remark finishes the proof of our Theorem 1.2.

\section{Asymptotic coefficients of minimal entire solu- tions and the basic properties of these solutions and of the corresponding monodromy matrices}

Choose two canonical bases $f_{1,2}$ and $g_{1,2}$. Consider the corresponding minimal solutions. Assume that these solutions exist and that their asymptotic coefficients are non zero. In this section, we study some basic properties of the minimal solutions and of the corresponding monodromy matrices. We shall use the notations from the section "Asymptotic coefficients" of the introduction.

\subsection{Wronskians of the minimal solutions}

Here, we study the wronskians of the minimal entire solutions and check Proposition 1.3. One has

Lemma 6.1. The wronskian of any two of the four minimal solutions corresponding to the bases $f_{1,2}$ and $g_{1,2}$ is independent of $z$.

Proof. Consider the minimal solutions $\psi_{D}$ and $\psi_{B}$. Study their wronskian. Remind that it is an $h$-periodic entire function.

In a vicinity of $+i \infty$,

$$
\begin{aligned}
\left\{\psi_{D}(z), \psi_{B}(z)\right\}= & \left\{A^{(D)}(z) f_{1}(z)+B^{(D)}(z) f_{2}(z), A^{(B)}(z) f_{1}(z)+B^{(B)}(z) f_{2}(z)\right\}= \\
& =w_{f}\left(A^{(D)}(z) B^{(B)}(z)-A^{(B)}(z) B^{(D)}(z)\right) .
\end{aligned}
$$


Here, we have marked by the letters $B$ and $D$ the functions $A(z)$ and $B(z)$ from the representations (19) for the solutions $\psi_{D}$ and $\psi_{B}$. The obtained formula for their wronskian implies that

$$
\left\{\psi_{D}(z), \psi_{B}(z)\right\} \rightarrow-w_{f} A_{B} B_{D}, \quad z \rightarrow+i \infty .
$$

Similarly, one proves that

$$
\left\{\psi_{D}(z), \psi_{B}(z)\right\} \rightarrow w_{g} C_{D} D_{B}, \quad z \rightarrow-i \infty
$$

Thus, the wronskian is a bounded entire function, and, therefore, it is independent of $z$.

The cases of the other pairs of the minimal solutions can be treated in the same way.

Asymptotics (156) and (157) imply the formulae

$$
\left\{\psi_{D}, \psi_{B}\right\}=w_{g} C_{D} D_{B}=-w_{f} A_{B} B_{D}
$$

In fact, this is two of the formulae described in Proposition 1.3. The other formulae can be derived similarly.

\subsection{Uniqueness of the minimal entire solutions}

Here, we deduce from Proposition 1.3 its Corollary 1.5. It suffices to check

Lemma 6.2. Consider two of the minimal solutions corresponding to the canonical bases $f_{1,2}$ and $g_{1,2}$. If their wronskian is nonzero, then each of these solutions is unique up to an independent of $z$ factor.

Proof. Consider the case of the solutions $\psi_{D}$ and $\psi_{B}$. The other cases can be treated similarly.

Let $\psi$ be a minimal solution for which, as for $\psi_{D}, D(z)$ tends to zero as $z \rightarrow-i \infty$. Show that $\psi(z)=$ Const $\psi_{D}(z)$. By Lemma 6.1 , the wronskian $w=\left\{\psi_{D}, \psi_{B}\right\}$ is independent of $z$, and by the assumption, it is nonzero. Thus, $\psi_{D}$ and $\psi_{B}$ form a base in the space of entire solutions of (1), and, so, $\psi$ can be represented in the form

$$
\psi(z)=\alpha(z) \psi_{D}+\beta(z) \psi_{B},
$$

where the coefficients $\alpha$ and $\beta$ are entire and $h$-periodic,

$$
\alpha(z)=\frac{1}{w}\left\{\psi(z), \psi_{B}(z)\right\}, \quad \beta(z)=\frac{1}{w}\left\{\psi_{D}(z), \psi(z)\right\} .
$$


Since all the three solutions are minimal solutions corresponding to one and the same pair of canonical bases, $\alpha$ and $\beta$ are constant. Let $C(z)$ and $D(z)$ be the periodic coefficients from the representations (20) for the solution $\psi$, and let $C^{(D)}(z)$ and $D^{(D)}(z)$ be the ones for the solution $\psi_{D}$. As in the proof of Lemma 6.1, we check that

$$
\beta(z)=\frac{w_{g}}{w}\left(C^{(D)}(z) D(z)-C(z) D^{(D)}(z)\right),
$$

in a vicinity of $-i \infty$. As $D(z)$ and $D^{(D)}(z)$ tend to zero as $z \rightarrow-i \infty$, formula (159) implies that $\beta \rightarrow 0$ as $z \rightarrow-i \infty$, and, thus, $\beta \equiv 0$. So, the minimal solution $\psi_{D}$ is unique up to a constant factor. In the same way, one proves the same statement for $\psi_{B}$.

q.e.d.

\subsection{Monodromy matrices corresponding to the minimal en- tire solutions}

We begin this subsection by proving Theorem 1.6.

Let

$$
w=\left\{\psi_{D}, \psi_{B}\right\}
$$

By the hypothesis of the theorem the wronskian $w$ is nonzero. It is given by formula (158).

The monodromy matrix corresponding to $\psi_{D}$ and $\psi_{B}$ is defined by

$$
\left(\psi_{D}(z+2 \pi), \psi_{B}(z+2 \pi)\right)=\left(\psi_{D}(z), \psi_{B}(z)\right) \mathcal{M}^{T}(z)
$$

The coefficients of the monodromy matrix admit the representations:

$$
\begin{aligned}
& \mathcal{M}_{11}(z)=\frac{1}{w}\left\{\psi_{D}(z+2 \pi), \psi_{B}(z)\right\}, \quad \mathcal{M}_{12}(z)=\frac{1}{w}\left\{\psi_{D}(z), \psi_{D}(z+2 \pi)\right\} \\
& \mathcal{M}_{21}(z)=\frac{1}{w}\left\{\psi_{B}(z+2 \pi), \psi_{B}(z)\right\}, \quad \mathcal{M}_{22}(z)=\frac{1}{w}\left\{\psi_{D}(z), \psi_{B}(z+2 \pi)\right\} .
\end{aligned}
$$

As in the proof of Lemma 6.1, one can easily calculate all the wronskians in (160) using the canonical bases $f_{1,2}$ and $g_{1,2}$. Consider, for example, the coefficient $\mathcal{M}_{11}$. By means of the representation (19), we get

$$
\begin{aligned}
\mathcal{M}_{11}=\frac{1}{w}\left(A ^ { ( D ) } ( z + 2 \pi ) A ^ { ( B ) } ( z ) \left\{f_{1}(z+2 \pi),\right.\right. & \left.f_{1}(z)\right\}+ \\
+A^{(D)}(z+2 \pi) B^{(B)}(z)\left\{f_{1}(z+2 \pi),\right. & \left.f_{2}(z)\right\}+B^{(D)}(z+2 \pi) A^{(B)}(z)\left\{f_{2}(z+2 \pi), f_{1}(z)\right\}+ \\
& \left.+B^{(D)}(z+2 \pi) B^{(B)}(z)\left\{f_{2}(z+2 \pi), f_{2}(z)\right\}\right) .
\end{aligned}
$$


Now, we recall that $f_{1,2}$ and $g_{1,2}$ are Bloch solutions,

$$
f_{1,2}(z+2 \pi)=\alpha_{1,2}(z) f_{1,2}(z), \quad g_{1,2}(z+2 \pi)=\beta_{1,2}(z) g_{1,2}(z)
$$

where $\alpha_{1,2}$ and $\beta_{1,2}$ are $h$-periodic functions. This allows to continue the calculation began in (161):

$$
\mathcal{M}_{11}=\frac{w_{f}}{w}\left(\alpha_{1}(z) A^{(D)}(z+2 \pi) B^{(B)}(z)-\alpha_{2}(z) B^{(D)}(z+2 \pi) A^{(B)}(z)\right) .
$$

The last formula allows to get the asymptotics of $\mathcal{M}_{11}$ for $z \rightarrow+i \infty$. Using the representation (16) and recalling that $n_{+}(v)=n$, we get

$$
\mathcal{M}_{11}=-\alpha_{2}^{0} \frac{w_{f}}{w} A_{B} B_{D} e^{-\frac{2 \pi i n}{h} z}(1+o(1)), \quad z \rightarrow+i \infty .
$$

Here, $A_{B}$ and $B_{D}$ are the asymptotic coefficients of $\psi_{B}$ and $\psi_{D}$. Now, using the formula (158) for $w$, we get finally:

$$
\mathcal{M}_{11}=\alpha_{2}^{0} e^{-\frac{2 \pi i n}{h} z}(1+o(1)), \quad z \rightarrow+i \infty .
$$

Remind that the constant $\alpha_{2}^{0}$ from (16) is nonzero.

Similarly, one can obtain

$$
\mathcal{M}_{11}=\beta_{1}^{0} e^{+\frac{2 \pi i n}{h} z}(1+o(1)), \quad z \rightarrow-i \infty
$$

with the nonzero constant $\beta_{1}^{0}$ from (17). Formulae (162) - (163) imply that the entire $h$-periodic function $\mathcal{M}_{11}$ is a trigonometric polynomial. Considering it as function of the variable $z_{1}=\frac{2 \pi}{h} z$, we see that

$$
n_{ \pm}\left(\mathcal{M}_{11}\right)=n
$$

Formulae (162) - (163) are, in fact, the first two of the formulae (25) - (32) for the coefficients of the monodromy matrix corresponding to the solutions $\psi_{D}$ and $\psi_{B}$. In the same way, one investigates all the other coefficients of the monodromy matrix. This leads to the formulae (25) - (32). For $\mathcal{M}$ considered as a function of $z_{1}=\frac{2 \pi}{h} z$, they obviously imply that

$$
\begin{gathered}
n_{-}\left(\mathcal{M}_{12}\right) \leq n-1, \quad n_{+}\left(\mathcal{M}_{12}\right) \leq n \\
n_{-}\left(\mathcal{M}_{21}\right) \leq n, \quad n_{+}\left(\mathcal{M}_{21}\right) \leq n-1 \\
n_{ \pm}\left(\mathcal{M}_{22}\right) \leq n-1
\end{gathered}
$$

These inequalities, and formulae (164) mean that the monodromy matrix, as a function of the variable $z_{1}=\frac{2 \pi}{h} z$, belongs to $\Omega(n)$. This completes the proof of the theorem. 
We finish this section by formulating one more theorem generalizing Theorem 1.6. As we have seen, any two of the minimal solutions form a basis in the space of entire solutions of (1). We call these bases natural. One has

Theorem 6.3. Let, the matrix $M$ satisfy condition (21). For any natural basis, the coefficients of the corresponding monodromy matrix $\mathcal{M}$ are trigonometric polynomials of the variable

$$
z_{1}=\frac{2 \pi}{h} z
$$

and for any of these polynomials the numbers $n_{ \pm}$satisfy the inequality

$$
-n \leq n_{ \pm} \leq n
$$

The proof of this theorem is similar to the proof of Theorem 1.6. It can be said that Theorem 6.3 describes the characteristic property of the minimal entire solutions.

\subsection{Canonical factorizations of the monodromy matrices}

For $M \in \Omega(n), n>1$, one can reconstruct in terms of the asymptotic coefficients of the minimal solutions only a part of the constant coefficients of the monodromy matrix. For example, formulae (25) - (26) allow to recover only the coefficients $\left(\mathcal{M}_{11}\right)_{ \pm n}$ of the trigonometric polynomial $\mathcal{M}_{11}$,

$$
\begin{aligned}
\mathcal{M}_{11}(z)=\left(\mathcal{M}_{11}\right)_{-n} e^{-2 \pi i n z / h}+\left(\mathcal{M}_{11}\right)_{-n+1} e^{-2 \pi i(n-1) z / h_{+}} & \\
& +\ldots+\left(\mathcal{M}_{11}\right)_{n} e^{2 \pi i n z / h} .
\end{aligned}
$$

Trying to get an efficient description of the other coefficients, we come to the following construction.

Let $f_{1,2}$ and $g_{1,2}$ be two consistent canonical bases with the parameters $\phi_{ \pm}$. Consider canonical bases $f_{1,2}^{(j)}$ and $g_{1,2}^{(j)}$ with the parameters equal to $\phi_{ \pm}+2 \pi j, j=$ $0,1,2, \ldots n$. Note that all the pairs $f_{1,2}^{(j)}$ and $g_{1,2}^{(j)}$ are consistent. By definition,

$$
f_{1,2}^{(0)}=f_{1,2}, \quad g_{1,2}^{(0)}=g_{1,2},
$$

In view of the analysis of section 2.5.2, we can assume that

$$
f_{1,2}^{(n)}(z)=s_{+} f_{1,2}(z+2 \pi), \quad g_{1,2}^{(n)}(z)=s_{-} g_{1,2}(z+2 \pi),
$$

where $s_{ \pm}$are two numbers, each of them can be equal either to +1 or to -1 . For the sake of definitiness, we consider the case where $s_{+}=s_{-}=1$. 
Denote by $\psi_{D}^{(j)}$ and $\psi_{B}^{(j)}$ the minimal solutions corresponding to the canonical bases $f_{1,2}^{(j)}$ and $g_{1,2}^{(j)}$. Let

$$
\psi_{D}=\psi_{D}^{(0)}, \quad \psi_{B}=\psi_{B}^{(0)}
$$

In view of Lemma 6.2 , we can assume that

$$
\psi_{D}^{(n)}(z)=\psi_{D}(z+2 \pi), \quad \psi_{B}^{(n)}(z)=\psi_{B}(z+2 \pi)
$$

Let, for any $j=0,1,2, \ldots n-1$, the solutions $\psi_{D}^{(j)}$ and $\psi_{B}^{(j)}$ be linearly independent over the ring of $h$-periodic functions.

Denote by $\Psi^{(j)}$ the $2 \times 2$-matrix composed of the vectors $\psi_{D}^{(j)}$ and $\psi_{B}^{(j)}$. Any of the pairs $\psi_{D}^{(j)}$ and $\psi_{B}^{(j)}$ is a basis in the space of entire solutions of (1). Therefore, one can define the transition matrices $S_{j}(z)$ relating $\Psi^{(j)}$ to $\Psi^{(j-1)}$,

$$
\Psi^{(j)}(z)=\Psi^{(j-1)}(z) S_{j}^{t}(z)
$$

where "t " denotes the transposition.

The monodromy matrix corresponding to the basis $\psi_{D}, \psi_{B}$ is represented in the form

$$
\mathcal{M}(z)=S_{n-1}(z) S_{n-2}(z) \ldots S_{1}(z) .
$$

We call this factorization of the monodromy matrix canonical. The main feature of this factorization is related to the standard (canonical) form of the transition matrices $S_{j}$. One has

Theorem 6.4. Any of the transitions matrices is an h-periodic trigonometric polynomial of the form

$$
\left(\begin{array}{cc}
a_{1} e^{2 \pi i z / h}+a_{0}+a_{-1} e^{-2 \pi i z / h} & b_{0}+b_{-1} e^{-2 \pi i z / h} \\
c_{0}+c_{-1} e^{2 \pi i z / h} & d_{0}
\end{array}\right)
$$

where $a_{ \pm 1,0}, b_{-1,0}, c_{1,0}$ and $d_{0}$ are constant coefficients (depending on $j$ ), and $a_{ \pm} \neq$ 0 . The $\operatorname{det} S_{j}$ are nonzero and independent of $z$.

The theorem shows that any of the transition matrices coincides up to a constant factor with a matrix from $\Omega(1)$. The proof of this theorem is similar to one of Theorem 1.6. The constant coefficients of $S_{j}$ can be expressed in terms of the asymptotic coefficients of the minimal solutions $\psi_{D}^{(j)}, \psi_{B}^{(j)}$, and $\psi_{D}^{(j-1)}, \psi_{B}^{(j-1)}$ by formulae similar to $(25)-(32)$. 


\section{One dimensional difference Schrödinger equa- tions}

Consider the difference Schrödinger equation

$$
\frac{f(z+h)+f(z-h)}{2}+v(z) f(z)=E f(z)
$$

where $v$ is a given trigonometric polynomial, and $E$ is a spectral parameter. It is equivalent to equation (1) with the matrix

$$
M=\left(\begin{array}{cc}
2 E-2 v(z) & -1 \\
1 & 0
\end{array}\right) .
$$

Assume that $n_{+}(v)=n_{-}(v)=n \in \mathbb{N}$. Then, this matrix belongs to the set $\Omega_{n n}$. Thus, one can apply Theorem 1.6 to investigate the spectrum of (165) by means of the monodromization procedure.

In this section, we concentrate on the Harper equation

$$
\frac{f(x+h)+f(x-h)}{2}+\lambda \cos z f(z)=E f(z),
$$

in which, $\lambda$ is a fixed positive parameter. Let us study for this equation the monodromy matrix described in Theorem 1.6 in more detail.

\subsection{Monodromy matrices for Harper equation}

1. First, discuss the choice of the bases $f_{1,2}$ and $g_{1,2}$. The formulae (11) and (14) allow to choose the corresponding parameters $\phi_{ \pm}$so that

$$
\phi_{-}=-i \xi-\pi, \quad \phi_{+}=i \xi-\pi, \quad \xi=\ln \lambda \in \mathbb{R} .
$$

Since $\xi \in \mathbb{R}$, these canonical bases are consistent, and there exist all the four minimal entire solutions.

2. Consider the minimal solutions $\psi_{D}$ and $\psi_{B}$ corresponding to these canonical bases. Assume that these solutions are linearly independent.

The solutions $\psi_{D}$ and $\psi_{B}$ are defined up to independent of $z$ factors. Describe a convenient choice of these factors. Since the wronskian $\left\{\psi_{D}, \psi_{B}\right\} \neq 0$, the asymptotic coefficient $C_{D}$ is nonzero, see Proposition 1.3. Normalize the solution $\psi_{D}$ by dividing it by its asymptotic coefficient $C_{D}$. We use for the new minimal solution and its asymptotic coefficients the old notations. In particular, now, $C_{D} \equiv 1$.

3. To fix uniquely the second minimal solution, we note that the matrix (166) with $v=\cos z$ satisfies the relation

$$
M(2 \pi-z)=\sigma M^{-1}(z) \sigma, \quad \sigma=\left(\begin{array}{cc}
0 & 1 \\
1 & 0
\end{array}\right) .
$$


This implies that the vector

$$
\psi(z)=\sigma \psi_{D}(2 \pi-z+h)
$$

satisfies (1) together with $\psi_{D}$. Show that, up to a constant factor, $\psi$ coincides with $\psi_{B}$. Begin with

Lemma 7.1 For $z$ being in a vicinity of $-i \infty$,

$$
\sigma f_{1}(2 \pi-z+h)=-g_{1}(z) c_{1}(z), \quad \sigma f_{2}(2 \pi-z+h)=-g_{2}(z) c_{2}(z)
$$

where $c_{1}$ and $c_{2}$ are analytic h-periodic functions tending to 1 as $z \rightarrow \pm i \infty$.

Proof. Let us prove the first of the above relations. Note that, for the matrix (166) corresponding to Harper equation, the general asymptotic formulae for $f_{1}$ and $g_{1}$ take the form

$$
f_{1}(z)=e^{+\frac{i}{2 h}(z-\pi+i \xi)^{2}+\frac{i z}{2}}\left(\begin{array}{c}
1+o(1) \\
-\lambda e^{-i z}(1+o(1))
\end{array}\right)
$$

$$
\operatorname{Im} z \rightarrow+\infty
$$

and

$$
g_{1}(z)=e^{+\frac{i}{2 h}(z-\pi-i \xi)^{2}-\frac{i z}{2}}\left(\begin{array}{c}
1+o(1) \\
-\frac{1}{\lambda} e^{-i z+i h}(1+o(1))
\end{array}\right)
$$

$\operatorname{Im} z \rightarrow-\infty$.

Substituting (171) in the expression $-\sigma f_{1}(2 \pi-z+h)$, we check that it does have the representation (172). Moreover, one can easily see that if $f_{1}$ is a Bloch solution, then $\sigma f_{1}(2 \pi-z+h)$ is also a Bloch solution of (1). Now, Lemma 2.6 implies the desired result. The second relation can be derived similarly.

q.e.d.

This lemma immediately leads to

Lemma 7.2. The solution $\psi$ coincide with $\psi_{B}$ up to a constant factor.

Proof. The representations (19) for $\psi_{D}$ imply that, in a vicinity $\mathbb{C}_{-}$of $-i \infty$,

$$
\psi(z)=A^{(D)}(2 \pi-z+h) \sigma f_{1}(2 \pi-z+h)+B^{(D)}(2 \pi-z+h) \sigma f_{2}(2 \pi-z+h) .
$$

Therefore, in view if Lemma 7.1,

$$
\psi(z)=C(z) g_{1}(z)+D(z) g_{2}(z)
$$


where

$$
C(z)=-c_{1}(z) A^{(D)}(2 \pi-z+h), \quad D(z)=-c_{2}(z) B^{(D)}(2 \pi-z+h) .
$$

Similarly, one shows that in a vicinity $\mathbb{C}_{+}$of $+i \infty$,

$$
\psi(z)=A(z) f_{1}(z)+B(z) f_{2}(z),
$$

where

$$
\begin{aligned}
& A(z)=-c_{1}^{-1}(2 \pi-z+h) C^{(D)}(2 \pi-z+h), \\
& B(z)=-c_{2}^{-1}(2 \pi-z+h) D^{(D)}(2 \pi-z+h) .
\end{aligned}
$$

These representations show that $\psi$ is a minimal solution for which $B(z) \rightarrow 0$ as $z \rightarrow+i \infty$. Therefore, by Corollary 1.5 , it coincides with $\psi_{B}$ up to a constant factor.

4. In the sequel, we shall normalize the solution $\psi_{B}$ so that

$$
\psi_{B}(z)=\sigma \psi_{D}(2 \pi-z+h) .
$$

Note that, in this case, (173) and (175) imply the relations

$$
A_{B}=-C_{D}=-1, \quad B_{B}=-D_{D} e^{-4 \pi^{2} i / h}, \quad C_{B}=-A_{D}, \quad D_{B}=-B_{D} .
$$

5. Discuss now the monodromy matrix corresponding the chosen $\psi_{D}$ and $\psi_{B}$.

Theorem 7.3. The constructed minimal entire solutions $\psi_{D}$ and $\psi_{B}$ and their asymptotic coefficients are meromorphic in $E$,

$$
\left\{\psi_{D}, \psi_{B}\right\} \not \equiv 0
$$

and the corresponding monodromy matrix has the form

$$
\mathcal{M}=\left(\begin{array}{cc}
a-2 \lambda_{1} \cos (2 \pi z / h) & s+t e^{-2 \pi i z / h} \\
-s-t e^{2 \pi i z / h} & \frac{1}{\lambda_{1}} s t
\end{array}\right)
$$

where

$$
a=\lambda_{1} \frac{1-s^{2}-t^{2}}{s t}, \quad \lambda_{1}=\lambda^{2 \pi / h}
$$

and

$$
s=-\lambda_{1} \frac{D_{D}}{B_{D}}, \quad t=-\lambda_{1} A_{D}
$$

Proof. First, prove that $\psi_{D}$ and $\psi_{B}$ and their asymptotic coefficients are meromorphic in $E$. Consider the solution $\psi_{D}$ before its normalization by the condition 
$C_{D}=1$. It can be constructed by means of the integral equation (92). For the matrix (166) with $v(z)=\cos z$, this equation takes the form

$$
\psi(z)=m(z)+E \tilde{K} \psi(z)
$$

where $K$ is the integral operator with the kernel

$$
\kappa(z, \zeta)=\frac{1}{i h} \theta(z, \zeta) \frac{[m(z) \tilde{m}(\zeta)-m(\zeta) \tilde{m}(z)]}{\{m, \tilde{m}\}} .
$$

This equation describes the first component of the vector $\psi_{D}$. The integral operator is compact in the suitable function space, and the solution $\psi_{D}$ together with $\psi$ appears to be meromorphic in $E$.

Furthemore, the solutions $f_{1,2}$ and $g_{1,2}$, being constructed as in Section 2, are entire in $E$.

Finally, the asymptotic coefficients of $\psi$ can be expressed in terms of the wronskians of the canonical basis solutions $f_{1,2}$ and $g_{1,2}$, and of $\psi$ itself, for example, $C$ is the zeroth Fourier coefficient of the function $\frac{\left\{\psi^{(1)}, g_{2}\right\}}{\left\{g_{1}, g_{2}\right\}}$. Thus, the asymptotic coefficients are meromorphic in $E$.

Note that we have already calculated these asymptotic coefficients for $E=0$ in Section 3: formulae (77) - (80) imply that these meromorphic functions are not identically zero.

All this shows that the normalized minimal solution $\psi_{D}$, the minimal solution $\psi_{B}$ related to it by (176), and the asymptotic coefficients of these solutions are meromorphic in $E$. Moreover, since the asymptotic coefficients of $\psi_{D}$ are nontrivial meromorphic functions, the wronskian $\left\{\psi_{D}, \psi_{B}\right\}$ is not identically zero.

Now obtain the representation (178) for the monodromy matrix corresponding to the chosen minimal solutions $\psi_{D}$ and $\psi_{B}$. First, consider the coefficient $\mathcal{M}_{11}$. By Theorem 1.6, it is a trigonometric polynomial of the form

$$
a_{-1} e^{-2 \pi i z / h}+a_{0}+a_{1} e^{2 \pi i z / h} .
$$

Formulae (25) - (26) imply that

$$
a_{-1}=\alpha_{2}^{0}, \quad a_{1}=\beta_{1}^{0},
$$

where $\alpha_{2}^{0}$ and $\beta_{1}^{0}$ are the first Fourier coefficients of the Bloch multipliers $\alpha_{2}$ and $\beta_{1}$ of the Bloch solutions $f_{2}$ and $g_{1}$,

$$
f_{2}(z+2 \pi)=\alpha_{2}(z) f_{2}(z), \quad g_{1}(z+2 \pi)=\beta_{1}(z) g_{1}(z) .
$$

The solution $g_{1}$ admits the representation (172) which implies that $\beta_{1}^{0}=-\lambda^{2 \pi / h}=$ $-\lambda_{1}$. Similarly, $\alpha_{2}^{0}=-\lambda_{1}$, and, thus,

$$
\mathcal{M}_{11}(z)=a_{0}-2 \lambda_{1} \cos (2 \pi z / h) .
$$


Formulae (27) - (32) and relations (177) imply the desired representations for $\mathcal{M}_{12}$, $\mathcal{M}_{21}$, and $\mathcal{M}_{22}$. Formula for the coefficient $a_{0}$ follows from these representations and the equality $\operatorname{det} \mathcal{M} \equiv 1$.

q.e.d.

\subsection{Family of matrices generated by Harper equation}

Fix a positive $\lambda$. Define a family $\mathbb{H}(\lambda) \subset \Omega(1)$ of matrix functions. This family consists of a two dimensional manifold $\mathbb{H}^{0}$ and four one dimensional linear manifolds $h_{0}^{ \pm}$and $h_{1}^{ \pm}$. The $\mathbb{H}^{0}$ is the set of the matrices $\mathcal{M}(., \lambda, w)$ defined by

$$
\mathcal{M}(z, \lambda, w)=\left(\begin{array}{cc}
a-2 \lambda \cos z & s+t e^{-i z} \\
-s-t e^{i z} & \frac{1}{\lambda} s t
\end{array}\right)
$$

where

$$
\begin{gathered}
a=\lambda \frac{1-s^{2}-t^{2}}{s t}, \\
w=(s, t) \in \mathbb{C}^{2}, \quad s, t \neq 0 .
\end{gathered}
$$

The $h_{0}^{ \pm}$is the set of the matrices

$$
M_{0}^{ \pm}(z, \lambda, a)=\left(\begin{array}{cc}
a-2 \lambda \cos z & \pm 1 \\
\mp 1 & 0
\end{array}\right), \quad a \in \mathbb{C}
$$

and $h_{1}^{ \pm}$is the set of the matrices

$$
M_{1}^{ \pm}(z, \lambda, a)=\left(\begin{array}{cc}
a-2 \lambda \cos z & \pm e^{-i z} \\
\mp e^{i z} & 0
\end{array}\right), \quad a \in \mathbb{C} .
$$

Apply Theorem 1.6 to equation (1) with a matrix $M \in \mathbb{H}$. Choose the bases $f_{1,2}$ and $g_{1,2}$ so that

$$
\phi_{-}=-i \xi-\pi-\frac{h}{2} n_{-}(b), \quad \phi_{+}=i \xi-\pi-\frac{h}{2} n_{+}(b), \quad \xi=\ln \lambda \in \mathbb{R}
$$

These canonical bases are consistent, and there exist all the four minimal entire solutions. Assume that the asymptotic coefficient $C_{D}$ is nonzero.

We normalize the solution $\psi_{D}$ by the condition $C_{D}=1$ and define $\psi_{B}$ by formula (176). Since the matrix $M$ satisfies the relation (169), $\psi_{B}$ is really the desired minimal solution.

Assume that $\psi_{D}$ and $\psi_{B}$ are linearly independent over the ring of $h$-periodic functions. Instead of Theorem 7.3, one can prove 
Theorem 7.4. As a function of the variable $2 \pi z / h$, the monodromy matrix $\mathcal{M}$ corresponding to the constructed minimal entire solutions belongs to $\mathbb{H}\left(\lambda_{1}\right)$ with

$$
\lambda_{1}=\lambda^{2 \pi / h}
$$

In particular, if $\mathcal{M} \in \mathbb{H}^{0}$, then it has the form

$$
\begin{gathered}
\mathcal{M}\left(2 \pi z / h, \lambda_{1}, w_{1}\right), \quad w_{1}=\left(s_{1}, t_{1}\right), \\
s_{1}=-\left(-i \frac{\sqrt{\lambda}}{s} e^{i h / 8}\right) \lambda_{1} \frac{D_{D}}{B_{D}}, \quad t_{1}=-\left(-i \frac{\sqrt{\lambda}}{t} e^{i h / 8}\right) \lambda_{1} \frac{A_{D}}{C_{D}} .
\end{gathered}
$$

\section{References}

[1] J.Sjostrand B.Helffer. Analyse semi-classique pour l'équation de Harper. Mémoire de la SMF, 34, 1988.

[2] V.Firsanov M.Bobrovnikov. Wave diffraction in angular domains (in russian). Tomsk State University, Tomsk, 1988.

[3] A. Fedotov V. Buslaev. Monodromization and Harper equation. In Equations aux dérivées partielles, pages XXI-1 - XXI-21, Paris, France, 1994. Ecole Polytechnique.

[4] A. Fedotov V. Buslaev. Bloch solutions of difference equations. Algebra and Analysis (in russian, engl. translation in St.Petersburg Jour. of Math.), 7(4):74-121, 1995.

[5] A. Fedotov V. Buslaev. Spectral properties of the monodromy matrix for harper equation. In Équations aux dérivées partielles (Saint-Jean-de-Monts), pages IV-1 - IV-11, Paris, France, 1997. Ecole Polytechnique.

[6] A. Fedotov V. Buslaev. Minimal solutions of difference equations with periodic coefficients. In XIIth International Congress of Mathematical Physics, pages 303-308, Boston, 1999. International Press.

[7] A.Fedotov V.Buslaev. The functional structure of a monodromy matrix for Harper equation. Operator Theory: Advances and Applications, 70:321-342, 1994.

[8] A.Fedotov V.Buslaev. Harper equation: Monodromization without semiclassics. Algebra and Analysis (in russian, engl. translation in St.Petersburg Jour. of Math.), 8(2):75-109, 1996.

[9] A.Fedotov V.Buslaev. Difference equations with periodic coefficients. Prépublications Mathématiques 97-18, Université Paris Nord, Paris, France, 1997.

[10] M. Wilkinson. Critical properties of electron eigenstates in incommensurate systems. Proc. Royal Society of London. A, 391:305-350, 1984. 